\title{
Anomalous elasticity of nematic and critically soft elastomers
}

\author{
Olaf Stenull and T. C. Lubensky \\ Department of Physics and Astronomy \\ University of Pennsylvania \\ Philadelphia, PA 19104 \\ $U S A$
}

(Dated: August 20, 2018)

\begin{abstract}
Uniaxial elastomers are characterized by five elastic constants. If their elastic modulus $C_{5}$ describing the energy of shear strains in planes containing the anisotropy axis vanishes, they are said to be soft. In spatial dimensions $d$ less than or equal to 3 , soft elastomers exhibit anomalous elasticity with certain length-scale dependent bending moduli that diverge and shear moduli that vanish at large length-scales. Using renormalized field theory at $d=3$ and to first order in $\varepsilon=3-d$, we calculate critical exponents and other properties characterizing the anomalous elasticity of two soft systems: (i) nematic elastomers in which softness is a manifestation of a Goldstone mode induced by the spontaneous symmetry breaking associated with a transition from an isotropic state to a nematic state and (ii) a particular version of what we call a critically soft elastomer in which $C_{5}=0$ corresponds to a critical point terminating the stability regime of a uniaxial elastomer with $C_{5}>0$.
\end{abstract}

PACS numbers: 61.41.+e, 64.60.Fr, 64.60.Ak

\section{INTRODUCTION}

Liquid-crystalline elastomers [1, 2, 3] are elastic media with the macroscopic symmetry properties of liquid crystals [4, 5]. They consist of weakly crosslinked polymeric networks with mesogenic units. The existence of the rubbery crosslinked network has apparently little impact on liquid crystalline phase behavior. In fact, the usual thermotropic liquid crystal phases, i.e., the nematic, cholesteric, smectic- $A$, and smectic- $C$ phases have their elastomeric counterparts [3, 6]. However, because liquid-crystalline elastomers cannot flow, they have mechanical properties that differ significantly from standard liquid crystals. Usually, liquid-crystalline elastomers are prepared by crosslinking side-chain [10] or main-chain 7] polymers. Alternative methods of synthesis include the polymerization of monomeric solutes in a liquid-crystalline solvent [8] or the confinement of a conventional liquid crystal in a dilute flexible matrix such as aerosil [9].

The main subject of this paper are nematic liquidcrystalline elastomers, or briefly, nematic elastomers (NEs). For recent reviews on NEs see Refs. 10, 11]. These materials have unique properties that make them candidates for device applications. Temperature change [12] or illumination [13] can alter the orientational order and cause the elastomer to extend or contract as much as $400 \%$ [14]. This qualifies nematic elastomers as contestants for use in artificial muscles 15, 16]. Another striking property of nematic elastomers is their soft elasticity [17, 19, 20, 21] characterized by vanishing shear stresses for a range of longitudinal strains applied perpendicular to the uniaxial direction.

The origin of soft elasticity in NEs is the spontaneous breaking of rotational symmetry of the isotropic state induced by the development of orientational order in the nematic state [17, 18]. This spontaneous symme- try breaking has the consequence that NEs are not like conventional uniaxial elastomers characterized by five independent shear moduli. Rather, the elastic constant associated with shear in planes containing the anisotropy axis (customarily called $C_{5}$ ) vanishes in NEs.

Though we are primarily interested in NEs, we also study a particular version of another class of soft uniaxial elastomers in which the elastic constant $C_{5}$ simply vanishes for energetic or entropic reasons. In this case, $C_{5}=0$ is a critical point marking the boundary between the high-symmetry uniaxial phase with $C_{5}>0$ and a lowsymmetry sheared phase with $C_{5}<0$. In other words, $C_{5}$ acts like the temperature variable in a standard thermal phase transition. A complete model of this critical point requires the introduction of third and fourth order terms in the nonlinear strains to stabilize the system when $C_{5}<0$. Since this complete model is characterized by a large number of parameters, it is quite complex. Rather than analyze this full model, we consider simpler model systems, which we call critically soft elastomers or CSEs, defined by simply setting $C_{5}=0$ in the standard elastic energy of a uniaxial medium containing only quadratic terms in nonlinear strains. Remarkably, CSEs exhibit well-defined anomalous elasticity much like that of the more physical NEs even though they lack the nonlinear terms needed to stabilize their low-symmetry phase. CSEs are simpler in many ways than NEs, and the analysis of their anomalous behavior provides a useful and instructive tutorial prelude to the analysis of NEs.

On the level of mean-field theory, the elastic energies of NEs and CSEs coincide. Therefore, their respective elastic properties are equivalent above their mutual upper critical dimension 3 . For dimensions $d \leq 3$, however, fluctuations become important. These fluctuations lead to Grinstein-Pelcovits 22] type renormalizations culminating in anomalous elasticity, i.e., in a length-scale dependence of certain elastic constants, with different uni- 
versality classes for NEs and CSEs.

In the present paper we explore the anomalous elasticity of NEs and CSEs by carrying out a renormalization group (RG) analysis. Using the methods of renormalized field theory [23] we examine the scaling behavior of the elastic constants in $d=3$ as well as in $d=3-\varepsilon$ dimensions. A brief account of our work on NEs appears in Ref. [24]. Our work on CSEs has not been reported hitherto. We will treat only systems in which random stresses are not important. Random stresses lead to a different universality class with anomalous behavior [17, 25] below five rather than three dimensions.

The plan of presentation is as follows: In Sec. III we give a brief summary of our main results. In Sec. III we discuss CSEs. In Sec IIIA we briefly review some elements of the Lagrangian theory of elasticity and then set up a Landau-Ginzburg-Wilson elastic energy functional (Hamiltonian) for CSEs. As a prelude to the subsequent RG analysis we analyze the symmetry contents of this Hamiltonian. Section IIIB contains the core of our renormalized field theory of CSEs. We explain our diagrammatic perturbation calculation and its renormalization. By solving the appropriate RG equation, we ascertain the scaling behavior of displacement correlation functions and ultimately that of the relevant elastic moduli. We conclude Sec. III by making contact with conventional uniaxial elastomers by incorporating a small but nonvanishing $C_{5}$, which leads to semi-soft elasticity. Section IV deals with NEs and has an outline similar to that of Sec. III In Sec. IVA we derive a Landau-GinzburgWilson minimal model for NEs in form of a field theoretic Hamiltonian. Our renormalized field theory for this model is presented in Sec. IVB The main part of this paper concludes with Sec. $\nabla$ where we give concluding remarks. There are five Appendices. In Appendices \$ B] and [C] we derive Ward identities for CSEs and NEs. An alternative RG approach to NEs is sketched in Appendix $\mathrm{D}$ Appendix $\mathrm{E}$ contains details on the calculation of Feynman diagrams.

\section{SUMMARY OF RESULTS}

For the convenience of the reader we now summarize our main results before we get into details of our work.

\section{A. Critically soft elastomers}

The elastic constants of CSEs are defined via the model elastic energy

$$
\begin{aligned}
& \mathcal{H}=\frac{1}{2} \int d^{d_{\perp}} x_{\perp} \int d x_{d}\left\{C_{1} u_{d d}^{2}+K\left(\nabla_{\perp}^{2} u_{d}\right)^{2}\right. \\
& \left.+2 C_{2} u_{d d} u_{a a}+C_{3} u_{a a}^{2}+2 C_{4} u_{a b} u_{a b}+C_{5} u_{a d} u_{a d}\right\}
\end{aligned}
$$

where $u_{d}$ and $u_{a}, a=1, \ldots, d-1$, are, respectively, the directions parallel and perpendicular to the nematic order and $u_{d d}, u_{a b}$, and $u_{a d}$ are components of the Lagrangian nonlinear strain tensor [26, 27]. The elastic constant $C_{1}$ describes longitudinal shear along the anisotropy. $C_{2}$ couples strains along the anisotropy axis to shears in the plane perpendicular to the anisotropy axis. The elastic constants $C_{3}$ and $C_{4}$ are associated with shear purely in the plane perpendicular to the distinguished direction. $K$ is a bending modulus. The pure CSE system with soft elasticity is characterized by $C_{5}=0$. The coupling $C_{5}$ plays a role similar to that of the temperature in a thermal phase transition. When $C_{5}>0$, the system displays conventional uniaxial elasticity. When $C_{5}<0$, the system is unstable with respect to the formation of a lower-symmetry (sheared) elastic state. $C_{5}=0$ marks the transition between the two phases. At this point, the system shows critical behavior analogous to critical behavior at a thermal phase transition. Our simple CSE model does not include higher order terms in the strain to stabilize the sheared phase. We expect, however, that the full model with these terms included will have the same structure as our CSE model, at least for $C_{5} \geq 0$.

The inverse displacement correlation functions (vertex functions) obey scaling forms that can be expressed for $d<3$ as

$$
\begin{aligned}
\Gamma_{d d}\left(\mathbf{q}_{\perp}, q_{d}\right) & =\frac{K}{T} L_{\perp}^{-4} \ell^{4-\eta_{K}} \\
& \times \hat{\Phi}_{d d}\left(\frac{L_{\perp} \mathbf{q}_{\perp}}{\ell^{1}}, \frac{L_{d} q_{d}}{\ell^{\phi}}, \frac{C_{5}}{K} \frac{L_{\perp}^{2}}{\ell^{1 / \nu_{5}}}\right) \\
\Gamma_{a d}\left(\mathbf{q}_{\perp}, q_{d}\right) & =\frac{C_{2}}{T} L_{\perp}^{-1} L_{d}^{-1} \ell^{1+\eta_{2}+\phi} \\
& \times \hat{\Phi}_{a d}\left(\frac{L_{\perp} \mathbf{q}_{\perp}}{\ell^{1}}, \frac{L_{d} q_{d}}{\ell^{\phi}}, \frac{C_{5}}{K} \frac{L_{\perp}^{2}}{\ell^{1 / \nu_{5}}}\right) \\
\Gamma_{a b}\left(\mathbf{q}_{\perp}, q_{d}\right) & =\frac{C_{4}}{T} L_{\perp}^{-2} \ell^{2+\eta_{C}} \\
& \times \hat{\Phi}_{a b}\left(\frac{L_{\perp} \mathbf{q}_{\perp}}{\ell^{1}}, \frac{L_{d} q_{d}}{\ell^{\phi}}, \frac{C_{5}}{K} \frac{L_{\perp}^{2}}{\ell^{1 / \nu_{5}}}\right)
\end{aligned}
$$

where $q_{d}$ and $\mathbf{q}_{\perp}$ are, respectively, wavenumbers (or momenta) parallel and perpendicular to the anisotropy axis.

$$
\begin{aligned}
\eta_{K} & =4 \varepsilon / 7, \quad \eta_{C}=\varepsilon / 7, \quad \eta_{2}=\varepsilon / 7, \\
\nu_{5} & =1 / 2-\varepsilon / 28, \quad \phi=\left(4-\eta_{K}\right) / 2,
\end{aligned}
$$

where $\varepsilon=3-d$, are scaling exponents. In principle, the exponents $\phi$ and $\eta_{2}$ could be independent of the other exponents. For the CSE model, however, they are not at least to first order in $\varepsilon$. The scaling functions $\hat{\Phi}_{d d}\left(\mathbf{q}_{\perp}, q_{d}, e\right), \hat{\Phi}_{a d}\left(\mathbf{q}_{\perp}, q_{d}, e\right)$, and $\hat{\Phi}_{a b}\left(\mathbf{q}_{\perp}, q_{d}, e\right)$ are respectively proportional to $\mathbf{q}_{\perp}^{4}+q_{d}^{2}+e \mathbf{q}_{\perp}^{2}, \mathbf{q}_{\perp} q_{d}$ and $\mathbf{q}_{\perp}^{2}$ in the long-wavelength limit in mean-field theory. $\hat{\Phi}_{a b}$ also has a term proportional to $q_{d}^{4}$, but its coefficient is irrelevant and we will not be concerned with it here. The boundary between scaling and Gaussian behavior is 
marked by the non-linear length scales

$$
\begin{aligned}
L_{\perp} & \sim\left[\sqrt{C_{1} K^{3}} /\left(C_{4} T\right)\right]^{1 / \varepsilon}, \\
L_{d} & =\sqrt{C_{1} / K} L_{\perp}^{2},
\end{aligned}
$$

where $T$ is the temperature measured in units so that the Boltzmann constant is equal to 1 .

The above scaling forms imply the following scaling for the elastic constants:

$$
\begin{aligned}
C_{2} & \sim C_{3} \sim C_{4} \\
& \sim\left\{\begin{array}{lll}
\left(L_{\perp}\left|\mathbf{q}_{\perp}\right|\right)^{\eta_{C}} & \text { if } \xi_{5}\left|\mathbf{q}_{\perp}\right| \gg 1, q_{d}=0 \\
\left(L_{d} q_{d}\right)^{\eta_{C} / \phi} & \text { if } & \xi_{5}\left|\mathbf{q}_{\perp}\right| L_{d} / L_{\perp} \gg 1, \mathbf{q}_{\perp}=\mathbf{0} \\
\left(L_{\perp} \xi_{5}^{-1}\right)^{\eta_{C}} & \text { if } \quad\left(\mathbf{q}_{\perp}, q_{d}\right)=(\mathbf{0}, 0)
\end{array}\right.
\end{aligned}
$$

and

$$
K \sim\left\{\begin{array}{lll}
\left(L_{\perp}\left|\mathbf{q}_{\perp}\right|\right)^{-\eta_{K}} & \text { if } \xi_{5}\left|\mathbf{q}_{\perp}\right| \gg 1, q_{d}=0 \\
\left(L_{d} q_{d}\right)^{-\eta_{K} / \phi} & \text { if } \xi_{5}\left|\mathbf{q}_{\perp}\right| L_{d} / L_{\perp} \gg 1, \mathbf{q}_{\perp}=\mathbf{0} \\
\left(L_{\perp} \xi_{5}^{-1}\right)^{-\eta_{K}} & \text { if }\left(\mathbf{q}_{\perp}, q_{d}\right)=(\mathbf{0}, 0)
\end{array}\right.
$$

where $\xi_{5}$ is a correlation length given by

$$
\xi_{5}=L_{\perp}\left(L_{\perp}^{2} C_{5} / K\right)^{-\nu_{5}}
$$

The elastic constant $C_{1}$ is not renormalized and it is not singular in either the wavenumbers or $\xi_{5}$. When $C_{5}$ is nonzero, $\Gamma_{d d} \sim C_{5}^{\gamma_{5}} q_{\perp}^{2}+K q_{\perp}^{4}$ at small $\mathbf{q}$, where $\gamma_{5}=$ $\left(2-\eta_{K}\right) \nu_{5}$ and $K$ is given by the last expression in Eq. (2.6).

At exactly three dimensions the above power law singularities become logarithmic singularities

$$
\begin{aligned}
C_{2} & \sim C_{3} \sim C_{4} \sim\left|\ln \left(\left|\mathbf{q}_{\perp}\right| / \mu\right)\right|^{-1 / 7}, \\
K & \sim\left|\ln \left(\left|\mathbf{q}_{\perp}\right| / \mu\right)\right|^{4 / 7},
\end{aligned}
$$

where $\mu$ is a wavenumber scale. This logarithmic anomaly can be observed for $\xi_{\perp}\left|\mathbf{q}_{\perp}\right| \ll 1$, where

$$
\xi_{\perp}=\mu^{-1} \exp \left[32 \pi \sqrt{C_{1} K^{3}} /\left(7 T C_{4}\right)\right] .
$$

In the critical regime at small $C_{5}$, we find the following universal Poisson ratios:

$$
C_{2}^{2} /\left(C_{1} C_{4}\right)=0 \quad \text { and } \quad C_{3} / C_{4}=-1 / 2 .
$$

\section{B. Nematic elastomers}

At the transition from the isotropic to the nematic phase, liquid-crystalline elastomers undergo an anisotropic stretch relative to their isotropic reference state of a factor $\Lambda_{0 \|}$ along the nematic axis and $\Lambda_{0 \perp}$ perpendicular to it. In the absence of explicit uniaxial terms such as

$$
h \int d^{d_{\perp}} x_{\perp} \int d x_{d}\left[u_{d d}-\frac{1}{d} u_{i i}\right],
$$

the elastic energy of the nematic phase that forms spontaneously from an isotropic phase is soft, i.e., the elastic constant $C_{5}$ for shears in the uniaxial plane vanishes. After rescaling lengths measured relative to the anisotropic nematic reference state and displacements according to $x_{a} \rightarrow x_{a}, x_{d} \rightarrow \sqrt{r-1} x_{d}, u_{a} \rightarrow u_{a}$ and $u_{d} \rightarrow \sqrt{r-1} u_{d}$, where $r=\Lambda_{0||}^{2} / \Lambda_{0 \perp}^{2}$, the relevant parts of the elastic energy can be written when uniaxial terms are present and small as

$$
\begin{aligned}
\mathcal{H} & =\frac{1}{2} \int d^{d_{\perp}} x_{\perp} \int d x_{d}\left\{C_{1} v_{d d}^{2}+K\left(\nabla_{\perp}^{2} u_{d}\right)^{2}(2.1\right. \\
& \left.+2 C_{2} v_{d d} v_{a a}+C_{3} v_{a a}^{2}+2 C_{4} v_{a b}^{2}+C_{5} v_{a d}^{2}\right\},
\end{aligned}
$$

with nonstandard strains $v_{a b}=\frac{1}{2}\left(\partial_{a} u_{b}+\partial_{b} u_{a}-\right.$ $\left.\partial_{a} u_{d} \partial_{b} u_{d}\right), v_{d d}=\partial_{d} u_{d}+\frac{1}{2} \partial_{a} u_{d} \partial_{b} u_{d}$ and $v_{a d}=\frac{1}{2} \partial_{a} u_{d}$ and where $C_{5}$ goes linearly to zero with $h$ or the magnitude of other uniaxial terms.

The scaling of the inverse displacement correlation functions of NEs is similar to but not identical to that of the CSE model:

$$
\begin{aligned}
\Gamma_{d d}\left(\mathbf{q}_{\perp}, q_{d}\right) & =\frac{K}{T} L_{\perp}^{-4} \ell^{4-\eta_{K}} \\
& \times \hat{\Phi}_{d d}\left(\frac{L_{\perp} \mathbf{q}_{\perp}}{\ell^{1}}, \frac{L_{d} q_{d}}{\ell^{\phi}}, \frac{C_{5}}{K} \frac{L_{\perp}^{2}}{\ell^{1 / \nu_{h}}}, \frac{C_{1}}{C_{4}} \frac{1}{\ell^{\eta_{C}}}\right), \\
\Gamma_{a d}\left(\mathbf{q}_{\perp}, q_{d}\right) & =\frac{C_{2}}{T} L_{\perp}^{-1} L_{d}^{-1} \ell^{1+\phi} \\
& \times \hat{\Phi}_{a d}\left(\frac{L_{\perp} \mathbf{q}_{\perp}}{\ell^{1}}, \frac{L_{d} q_{d}}{\ell^{\phi}}, \frac{C_{5}}{K} \frac{L_{\perp}^{2}}{\ell^{1 / \nu_{h}}}\right), \\
\Gamma_{a b}\left(\mathbf{q}_{\perp}, q_{d}\right) & =\frac{C_{3}}{T} L_{\perp}^{-2} \ell^{2} \\
& \times \hat{\Phi}_{a b}\left(\frac{L_{\perp} \mathbf{q}_{\perp}}{\ell^{1}}, \frac{L_{d} q_{d}}{\ell^{\phi}}, \frac{C_{5}}{K} \frac{L_{\perp}^{2}}{\ell^{1 / \nu_{h}}}, \frac{C_{4}}{C_{3}} \ell^{\eta_{C}}\right),
\end{aligned}
$$

where, to first order in $\varepsilon$

$$
\begin{aligned}
& \eta_{K}=38 \epsilon / 59, \quad \eta_{C}=4 \epsilon / 59 \\
& \phi=2-21 \varepsilon / 59, \quad \nu_{h}=1 / 2+9 \varepsilon / 108
\end{aligned}
$$

and

$$
\begin{aligned}
L_{\perp} & \sim\left[\sqrt{K^{3} /\left(C_{4} T^{2}\right)}\right]^{1 / \varepsilon}, \\
L_{d} & =\sqrt{C_{4} / K} L_{\perp}^{2} .
\end{aligned}
$$

Note that four independent scaling exponents, $\eta_{K}, \eta_{C}$, $\phi$, and $\nu_{h}$ are required to describe NEs with a small uniaxial energy. In the above, lengths, displacements and $\mathbf{q}$-vectors are measured in rescaled units.

The above scaling forms predict that $C_{1}, C_{2}$, and $C_{3}$ are unrenormalized and that

$$
C_{4} \sim\left\{\begin{array}{lll}
\left(L_{\perp}\left|\mathbf{q}_{\perp}\right|\right)^{\eta_{C}} & \text { if } & \xi_{h}\left|\mathbf{q}_{\perp}\right| \gg 1, q_{d}=0 \\
\left(L_{d} q_{d}\right)^{\eta_{C} / \phi} & \text { if } & \xi_{h}\left|\mathbf{q}_{\perp}\right| L_{d} / L_{\perp} \gg 1, \mathbf{q}_{\perp}=\mathbf{0} \\
\left(L_{\perp} \xi_{h}^{-1}\right)^{\eta_{C}} & \text { if } \quad\left(\mathbf{q}_{\perp}, q_{d}\right)=(\mathbf{0}, 0)
\end{array}\right.
$$


as well as

$$
K \sim\left\{\begin{array}{lll}
\left(L_{\perp}\left|\mathbf{q}_{\perp}\right|\right)^{-\eta_{K}} & \text { if } \xi_{h}\left|\mathbf{q}_{\perp}\right| \gg 1, q_{d}=0 \\
\left(L_{d} q_{d}\right)^{-\eta_{K} / \phi} & \text { if } \quad \xi_{h}\left|\mathbf{q}_{\perp}\right| L_{d} / L_{\perp} \gg 1, \mathbf{q}_{\perp}=\mathbf{0} \\
\left(L_{\perp} \xi_{h}^{-1}\right)^{-\eta_{K}} & \text { if } \quad\left(\mathbf{q}_{\perp}, q_{d}\right)=(\mathbf{0}, 0)
\end{array}\right.
$$

with $\xi_{h}$ given by

$$
\xi_{h}=L_{\perp}\left(L_{\perp}^{2} h / K\right)^{-\nu_{h}}
$$

At small but nonzero $h, \Gamma_{d d} \sim h^{\gamma_{h}} q_{\perp}^{2}+K q_{\perp}^{4}$ at small q, where $\gamma_{h}=\left(2-\eta_{h}\right) \nu_{h}$ and $K$ is given by the last expression in Eq. (2.17).

At exactly three dimensions the above power laws become

$$
\begin{aligned}
C_{4} & \sim\left|\ln \left(\left|\mathbf{q}_{\perp}\right| / \mu\right)\right|^{-4 / 59}, \\
K & \sim\left|\ln \left(\left|\mathbf{q}_{\perp}\right| / \mu\right)\right|^{38 / 59} .
\end{aligned}
$$

The length scale that marks the crossover from harmonic to logarithmic behavior is

$$
\xi_{\perp}=\mu^{-1} \exp \left[64 \pi \sqrt{K^{3}} /\left(7 \sqrt{6 C_{4}} T\right)\right] .
$$

Provided that $C_{5}$ is small, the critical regime entails four independent Poisson ratios:

$$
\begin{aligned}
& C_{2} / C_{1}=1, \quad C_{3} / C_{1}=1, \quad C_{4} / C_{1}=0, \\
& \left(2 C_{2}-C_{3}-C_{1}\right) / C_{4}=1 / 2 .
\end{aligned}
$$

\section{CRITICALLY SOFT ELASTOMERS}

\section{A. The model}

We start by setting up a field theoretic minimal model for CSEs that is suitable for our subsequent RG analysis. We find it convenient to use the Lagrangian formulation of elasticity [26, 27]. In this formulation the mass points of the equilibrium undistorted medium are labeled by their position vectors $\mathbf{x}$ in $d$-dimensional (reference) space. When the medium is distorted, a mass point originally at $\mathbf{x}$ is mapped to a new point $\mathbf{R}(\mathbf{x})$ in $d$-dimensional (target) space. Since $\mathbf{R}(\mathbf{x})=\mathbf{x}$ when there is no distortion, it is customary to introduce the phonon variable $\mathbf{u}(\mathbf{x})=\mathbf{R}(\mathbf{x})-\mathbf{x}$ that measures the deviation of $\mathbf{R}(\mathbf{x})$ from $\mathbf{x}$.

Suppose for a moment the medium is distorted solely by stretching. The energy of the distorted state relative to the reference state depends on the relative amount of stretching $d \mathbf{R}^{2}-d \mathbf{x}^{2}=2 u_{i j} d x_{i} d x_{j}$, where

$$
u_{i j}=\frac{1}{2}\left\{\partial_{i} u_{j}+\partial_{j} u_{i}+\partial_{i} u_{k} \partial_{j} u_{k}\right\}
$$

with $i, j, k=1, \ldots, d[28]$ are the components of the familiar nonlinear Lagrangian strain tensor $\underline{u}$. Note that $\underline{\underline{u}}$ is invariant under arbitrary rotations in $\overline{\bar{t}}$ arget space.
This feature makes the Lagrangian strain tensor an adequate variable for formulating elastic energies because all elastic media are rotationally invariant in target space. This invariance is easy to understand: different physical orientations of the same sample have the same energy [29]. As customary, the reference state, relative to which $\underline{\underline{u}}$ is defined, is taken to be in mechanical equilibrium, and hence no terms linear in $u_{i j}$ appear in the stretching energy. To lowest order, the stretching energy is then of the form

$$
H_{\mathrm{st}}=\frac{1}{2} \int d^{d} x K_{i j k l} u_{i j} u_{k l},
$$

where $K_{i j k l}$ is an elastic constant tensor. For media isotropic in the reference space, for example, there are only two independent elastic constants in $K_{i j k l}$ that are known as the Lamé coefficients $\lambda$ and $\mu$. Media with uniaxial symmetry in the reference space are characterized in general by five independent elastic constants. Assuming that the anisotropy axis is in the $\hat{\mathbf{e}}_{d}=(0, \ldots, 1)$ direction we may write the stretching energy as

$$
\begin{aligned}
& H_{\mathrm{st}}=\frac{1}{2} \int d^{d_{\perp}} x_{\perp} \int d x_{d}\left\{C_{1} u_{d d}^{2}+2 C_{2} u_{d d} u_{a a}\right. \\
& \left.+C_{3} u_{a a}^{2}+2 C_{4} u_{a b}^{2}+C_{5} u_{a d}^{2}\right\}
\end{aligned}
$$

with $d_{\perp}=d-1$ and $a, b=1, \ldots, d_{\perp}$.

Now suppose that the elastic constant $C_{5}$ vanishes. Rewriting $H_{\text {st }}$ in Fourier space one sees easily that the stretching energy cost is zero for phonon displacements $\widetilde{\mathbf{u}}(\mathbf{q})$ perpendicular to $\hat{\mathbf{e}}_{d}$ with momentum $\mathbf{q}$ parallel to $\hat{\mathbf{e}}_{d}$ and for $\widetilde{\mathbf{u}}(\mathbf{q})$ parallel to $\hat{\mathbf{e}}_{d}$ with $\mathbf{q}$ perpendicular to $\hat{\mathbf{e}}_{d}$. In other words: CSEs are soft elastic materials.

For many elastic systems it is justified to neglect energetic contributions, such as bending, that are associated with higher derivatives of the displacements. That is, because bending is unimportant compared to stretching at small momenta. Due to the soft elasticity, however, the stretching energy of CSEs can vanish, and hence, bending is important.

For the moment, we set aside the uniaxial term proportional to $C_{5}$ and concentrate on the pure soft case. The effects of a small but non-vanishing $C_{5}$ will be included later on. Taking into account stretching and bending, the CSE model is defined by the Hamiltonian

$$
\begin{aligned}
& \mathcal{H}=\frac{1}{2} \int d^{d_{\perp}} x_{\perp} \int d x_{d}\left\{C_{1} u_{d d}^{2}+K\left(\nabla_{\perp}^{2} u_{d}\right)^{2}\right. \\
& \left.+2 C_{2} u_{d d} u_{a a}+C_{3} u_{a a}^{2}+2 C_{4} u_{a b} u_{a b}\right\}
\end{aligned}
$$

where $K$ is a bending modulus. All other bending terms allowed by symmetry turn out to be irrelevant in the sense of the renormalization group. Also, not all parts of the strains are relevant. Discarding any parts of the strains that lead to irrelevant contributions to the Hamiltonian, as discussed further below, leaves us with

$$
u_{a b}=\frac{1}{2}\left\{\partial_{a} u_{b}+\partial_{b} u_{a}+\partial_{a} u_{d} \partial_{b} u_{d}\right\}
$$


and

$$
u_{d d}=\partial_{d} u_{d} .
$$

In principle we could use $\mathcal{H}$ as it stands in Eq. (3.4) for our RG analysis. We find it convenient, however, to reduce the number of constants featured in $\mathcal{H}$ at the onset. To this end, we rescale $u_{a} \rightarrow\left(K / C_{4}\right) u_{a}, u_{d} \rightarrow$ $\sqrt{K / C_{4}} u_{d}$, and $x_{d} \rightarrow\left(C_{4} / K^{2}\right) x_{d}$. Then the Hamiltonian takes on the form

$$
\begin{aligned}
& \mathcal{H}=\frac{1}{2} \int d^{d_{\perp}} x_{\perp} \int d x_{d}\left\{\omega u_{d d}^{2}+\left(\nabla_{\perp}^{2} u_{d}\right)^{2}\right. \\
& \left.+2 g u_{d d} u_{a a}+f u_{a a}^{2}+2 u_{a b} u_{a b}\right\}
\end{aligned}
$$

where

$$
\omega=C_{1} K^{3} / C_{4}^{2}, \quad g=C_{2}\left(K / C_{4}\right)^{3 / 2}, \quad f=C_{3} / C_{4} .
$$

At this stage we would like to point out that we explicitly keep the temperature $T$ in the Boltzmann weight $\exp (-\mathcal{H} / T)$ 30] governing our field theoretic calculations. In what follows we carry out a perturbation expansion in the temperature, i.e., $T$ serves as our expansion parameter. As a consequence, not only the constants and fields featured in $\mathcal{H}$ but also the temperature will require renormalization.

An effective Hamiltonian for $u_{d}$ alone can be obtained by integrating out the transverse variable $u_{a}$ from the full CSE Hamiltonian of Eq. (3.4). When $C_{4}=\infty$, this process leads to the Hamiltonian for a smectic- $A$ liquid crystal whose anomalous elasticity was analyzed by Grinstein and Pelcovits 22]. Our rescaling of variables to obtain Eq. (3.6) with the coefficients of both $\left(\nabla_{\perp}^{2} u_{d}\right)^{2}$ and $2 u_{a b} u_{a b}$ set to unity is not ideally suited to taking the $C_{4}=\infty$ limit. Our primary interest is the anomalous elasticity unique to soft uniaxial systems for which the parametrization of Eq. (3.6) is appropriate. We will not give further consideration to the Grinstein-Pelcovits limit of our model.

As a further step towards our RG analysis, we now discuss the scaling symmetries of our model. First, under a global rescaling of the coordinates $x_{a} \rightarrow \mu^{-1} x_{a}$ and $x_{d} \rightarrow \mu^{-2} x_{d}$, we find a scaling invariant theory provided that $u_{a} \rightarrow \mu^{1} u_{a}$ and $T \rightarrow \mu^{3-d} T$ ( $\mu$ invariance). Viewing $\mu$ as an inverse length scale, this means that the field $u_{a}$ has a naive dimension 1 and that the naive dimension of $T$ is $\varepsilon=3-d$. The field $u_{d}$ and the remaining parameters in $\mathcal{H}$ have a vanishing naive dimension. Above $d=3$ dimensions the naive dimension of $T$ is negative and $T$ is irrelevant whereas it is relevant below $d=3$. Hence, we identify $d_{c}=3$ as the upper critical dimension of the CSE model.

At this point we take a short detour and catch up on justifying the truncation of the strains as stated in Eqs. (3.5). Applying the $\mu$-rescaling to the original full strains leads to

$$
u_{a b} \rightarrow \frac{\mu^{2}}{2}\left\{\partial_{a} u_{b}+\partial_{b} u_{a}+\partial_{a} u_{d} \partial_{b} u_{d}+\mu^{2} \partial_{a} u_{c} \partial_{b} u_{c}\right\}
$$

and

$$
u_{d d} \rightarrow \mu^{2}\left\{\partial_{d} u_{d}+\frac{\mu^{2}}{2}\left(\partial_{d} u_{d}\right)^{2}+\frac{\mu^{4}}{2} \partial_{d} u_{c} \partial_{d} u_{c}\right\} .
$$

The terms carrying extra powers of $\mu$ do not contribute to the leading behavior in the limit $\mu \rightarrow 0$. Hence, they can be neglected in studying the long length scale behavior at leading order, i.e., they are irrelevant in the sense of the RG.

Second, due to the anisotropy of the model, we may rescale the longitudinal coordinate alone: $x_{d} \rightarrow \beta x_{d}$. Scale invariance is retained if $\omega \rightarrow \beta^{2} \omega, g \rightarrow \beta g$, and $T \rightarrow \beta T$ ( $\beta$ invariance). Note that the composed couplings

$$
\sigma=g^{2} / \omega, \rho=f, \text { and } t=\mu^{-\varepsilon} T / \sqrt{\omega}
$$

are invariant under the longitudinal rescaling. The factor $\mu^{-\varepsilon}$ is included in the definition of $t$ to render it, like $\sigma$ and $\rho$, dimensionless. As we go along, we will see that $\sigma, \rho$, and $t$ emerge quite naturally in perturbation theory. Third, $\mathcal{H}$ is invariant under the rescaling $u_{d} \rightarrow u_{d}+f_{d}\left(\mathbf{x}_{\perp}\right)$, where $f_{d}$ is an arbitrary function of the transversal coordinate $\mathbf{x}_{\perp}$. Fourth, rescaling $u_{a} \rightarrow u_{a}+f_{a}\left(x_{d}\right)+M_{a b} x_{b}$ leaves $\mathcal{H}$ invariant if the matrix constituted by the $M_{a b}$ is antisymmetric and $f_{a}$ is a function of the longitudinal coordinate only. Finally, $\mathcal{H}$ is invariant under the transformation $u_{a} \rightarrow u_{a}+\theta_{d} u_{d}$ and $u_{d} \rightarrow u_{d}-\theta_{a} x_{a}$ provided that the $\theta$ 's are small. Note that this transformation mixes the longitudinal and the transversal fields (mixing invariance). It can be viewed as a remnant of the rotational invariance of the original theory in target space. This mixing transformation will be valuable for us because it leads to Ward identities that reduce the number of vertex functions to be calculated in perturbation theory. These Ward identities will be derived in Appendix $\mathbb{A}$

\section{B. Renormalization group analysis}

In this section we determine the scaling behavior of the correlation function of the fields $u_{a}(\mathbf{x})$ and $u_{d}(\mathbf{x})$ by using perturbation theory augmented by renormalization group methods. As usual, we analyze vertex functions that require renormalization due to the presence of ultraviolet (UV) divergences in Feynman diagrams. Our main tools in this section will be dimensional regularization and minimal subtraction. To avoid infrared (IR) singularities in the Feynman diagrams, we supplement our Hamiltonian with a mass term,

$$
\mathcal{H} \rightarrow \mathcal{H}+\frac{\tau}{2} \int d^{d_{\perp}} x_{\perp} \int d x_{d} u_{d}^{2} .
$$

At the appropriate stage of the calculations we then sent $\tau$ to zero to recover the original situation. 


\section{Diagrammatic expansion}

In order to set up a diagrammatic perturbation expansion we have to determine its constituting elements. First, we have the Gaussian propagator $G$ that has the form of a $d \times d$ matrix. The elements of its inverse $\underline{\underline{\underline{\Gamma}}}$ are readily collected from the Hamiltonian:

$$
\begin{aligned}
\Gamma_{d d} & =T^{-1}\left[\tau+\omega q_{d}^{2}+\mathbf{q}_{\perp}^{4}\right], \\
\Gamma_{a d} & =T^{-1} g q_{a} q_{d}, \\
\Gamma_{a b} & =T^{-1}\left[(f+1) q_{a} q_{b}+\delta_{a b} \mathbf{q}_{\perp}^{2}\right] .
\end{aligned}
$$

Inverting $\underline{\underline{\Gamma}}$ we find that the Gaussian propagator has the elements

$$
\begin{aligned}
G_{d d} & =T \frac{B}{B \tau+A q_{d}^{2}+B \mathbf{q}_{\perp}^{4}} \\
G_{a d} & =T \frac{-g}{B \tau+A q_{d}^{2}+B \mathbf{q}_{\perp}^{4}} \frac{q_{a} q_{d}}{\mathbf{q}_{\perp}^{2}} \\
G_{a b} & =T\left[\frac{\delta_{a b}}{\mathbf{q}_{\perp}^{2}}-\frac{D \tau+C q_{d}^{2}+D \mathbf{q}_{\perp}^{4}}{B \tau+A q_{d}^{2}+B \mathbf{q}_{\perp}^{4}} \frac{q_{a} q_{b}}{\mathbf{q}_{\perp}^{4}}\right]
\end{aligned}
$$

where we have used the shorthand notations $A=\omega(f+$ 2) $-g^{2}, B=f+2, C=\omega(f+1)-g^{2}$, and $D=f+$ 1. Second, our diagrammatic expansion features the 4 vertices

$$
\begin{aligned}
& i \frac{g}{2 T} q_{d}^{(1)} q_{b}^{(2)} q_{b}^{(3)}, \\
& i \frac{f}{2 T} q_{a}^{(1)} q_{b}^{(2)} q_{b}^{(3)}, \\
& i \frac{1}{T} q_{a}^{(2)} q_{b}^{(1)} q_{b}^{(3)}, \\
& -\frac{f+2}{8 T} q_{a}^{(1)} q_{a}^{(2)} q_{b}^{(3)} q_{b}^{(4)}
\end{aligned}
$$

It is understood that the sum of the momenta has to vanish at each vertex.

Next, we need to determine which of the vertex functions $\Gamma^{(M, N)}$ with $M$ external $u_{a}$-legs and $N$ external $u_{d}$-legs are superficially UV divergent. Analyzing their topology, we find that the superficial degree of divergence $\delta$ of our diagrams is given at the upper critical dimension by $\delta=4-M-2 D_{\|}-D_{\perp}$, where $D_{\|}\left(D_{\perp}\right)$ is the number of longitudinal (transversal) derivatives on the external legs. Thus, the only vertex functions containing superficially divergent diagrams are $\Gamma_{d}^{(0,1)}, \Gamma_{a}^{(1,0)}, \Gamma_{d d}^{(0,2)}$, $\Gamma_{a d}^{(1,1)}, \Gamma_{a b}^{(2,0)}, \Gamma_{d d d}^{(0,3)}, \Gamma_{a d d}^{(1,2)}$, and $\Gamma_{d d d d}^{(0,4)}$. All these vertex functions have to be taken into account in the renormalization procedure. By virtue of the mixing invariance, however, there exist several relations between the vertex functions in form of Ward identities. These are derived and stated in Appendix A Due to these Ward identities it is sufficient for our purposes to actually calculate the two-point functions $\Gamma_{d d}^{(0,2)}, \Gamma_{a d}^{(1,1)}$, and $\Gamma_{a b}^{(2,0)}$. Once the equations of state $\Gamma_{d}^{(0,1)}=0$ and $\Gamma_{a}^{(1,0)}=0$ are satisfied and the 2-point functions are renormalized, the Ward

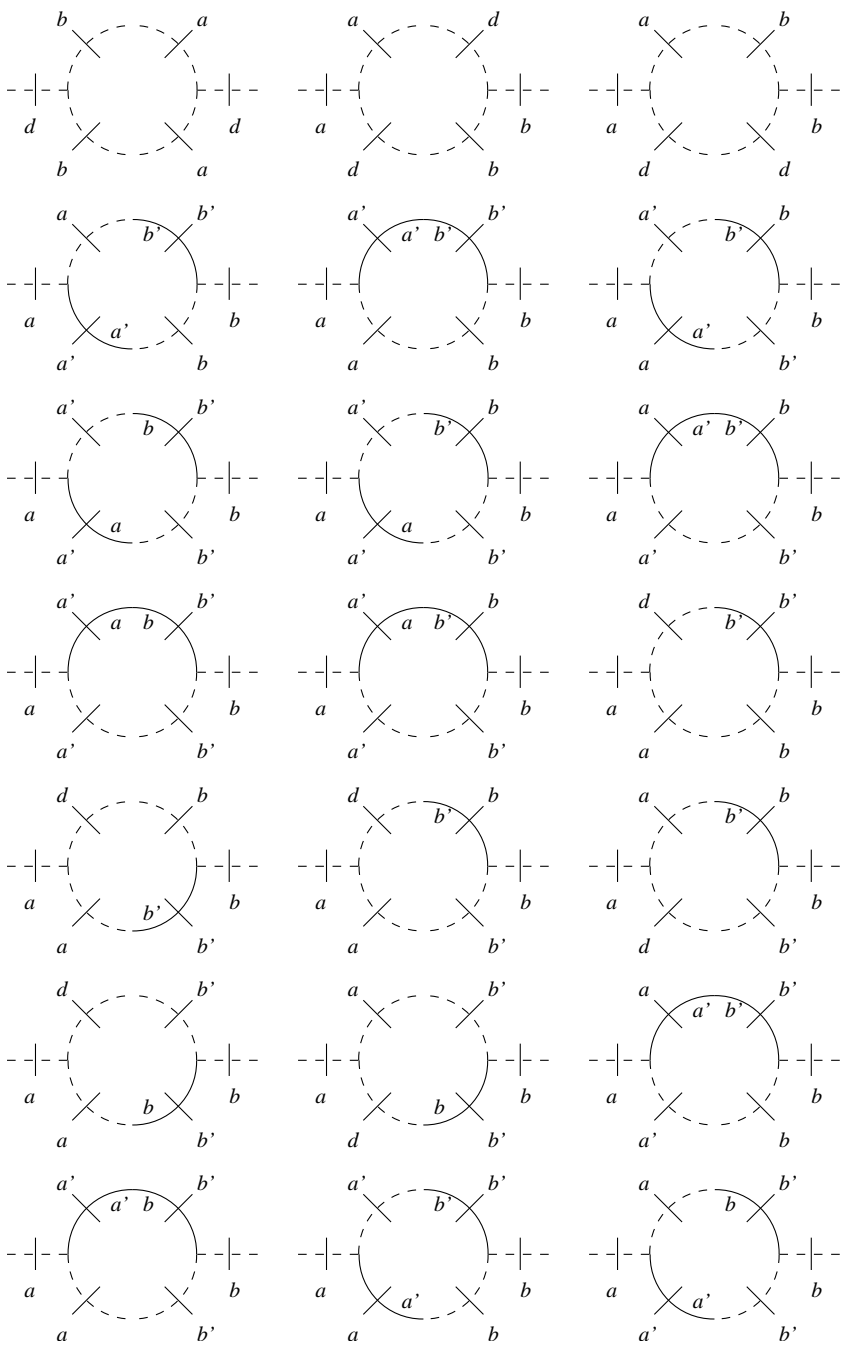

FIG. 1: Feynman diagrams contributing to $\Gamma_{d d}$. The dashed lines symbolize the element $G_{d d}$ of the Gaussian propagator. Lines half dashed and half solid with an index, say $a$, stand for $G_{a d}$. Solid lines with 2 indices, say $a$ and $b$, visualize $G_{a b}$. The ticks indicate derivatives with respect to the reference space coordinate with an index specifying the component.

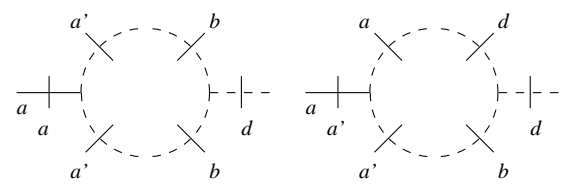

FIG. 2: Feynman diagrams contributing to $\Gamma_{a d}$. The meaning of the symbols is the same as in Fig. 1]

identities guarantee that the remaining vertex functions are cured of their UV divergences.

We calculate the two-point vertex functions to one-loop order using dimensional regularization. The Feynman diagrams entering this calculation are listed in Figs. 1 to 3 Details on computing the diagrams can be found in Appendix $\mathbb{E}$ Our results for the 2-point functions read 


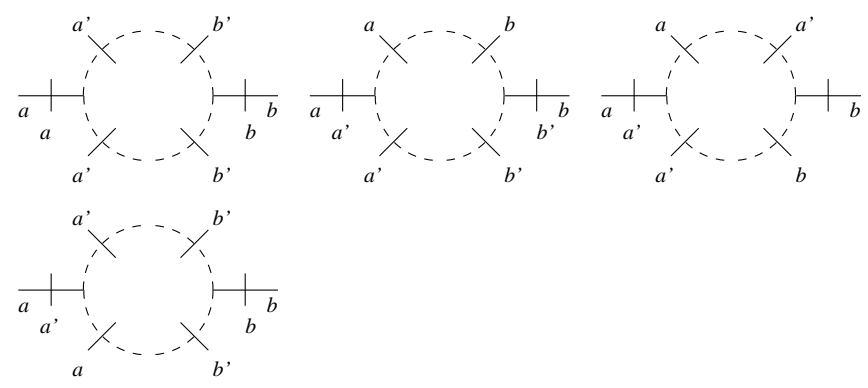

FIG. 3: Feynman diagrams contributing to $\Gamma_{a b}$. The meaning of the symbols is the same as in Fig. 1

$$
\begin{aligned}
& \Gamma_{d d}^{(0,2)}=T^{-1}\left[\tau+\omega q_{d}^{2}+\mathbf{q}_{\perp}^{4}\right] \\
& -\frac{\sqrt{2+f} g^{2} q_{d}^{2}}{16 \pi \varepsilon \sqrt{-g^{2}+(2+f) \omega}} \tau^{-\varepsilon / 4} \\
& -\frac{3(1+f) \mathbf{q}_{\perp}^{4}}{8 \pi \varepsilon \sqrt{2+f} \sqrt{-g^{2}+(2+f) \omega}} \tau^{-\varepsilon / 4}, \\
& \Gamma_{a d}^{(1,1)}=T^{-1} g q_{a} q_{d} \\
& -\frac{\sqrt{2+f}(1+f) g q_{d} q_{a}}{16 \pi \varepsilon \sqrt{-g^{2}+(2+f) \omega}} \tau^{-\varepsilon / 4} \\
& \Gamma_{a b}^{(2,0)}=T^{-1}\left[(f+1) q_{a} q_{b}+\delta_{a b} \mathbf{q}_{\perp}^{2}\right] \\
& -\frac{\sqrt{2+f}\left[2(1+f)^{2} q_{a} q_{b}+\delta_{a b} \mathbf{q}_{\perp}^{2}\right]}{32 \pi \varepsilon \sqrt{-g^{2}+(2+f) \omega}} \tau^{-\varepsilon / 4} .
\end{aligned}
$$

As already indicated by the vertex functions' superficial degree of divergence, higher order terms in the momentum expansion are convergent and hence can be neglected for our purposes. Likewise, contributions proportional to the mass $\tau$ are convergent. Thus, $\tau$ does not require renormalization and consequently its scaling dimension is identical to its naive dimension four. At this stage of the calculation $\tau$ has fulfilled its purpose, viz. it prevented IR singularities from producing spurious $\varepsilon$ poles. In conjunction with the $\varepsilon$ expansion we now can safely send $\tau$ to zero.

\section{Renormalization}

The UV divergences have their manifestation in the $\varepsilon$ poles appearing in Eqs. 3.14. We eliminate these poles by employing the renormalization scheme

$$
\begin{aligned}
& u_{d} \rightarrow \stackrel{\circ}{u}_{d}=Z^{1 / 2} u_{d}, \\
& u_{a} \rightarrow \stackrel{\circ}{u}_{a}=Z u_{a}, \\
& T \rightarrow \stackrel{\circ}{T}=Z Z_{T}^{-1} T, \\
& \omega \rightarrow \stackrel{\circ}{=}=Z_{T}^{-1} Z_{\omega} \omega, \\
& g \rightarrow \stackrel{\circ}{=}=Z^{-1 / 2} Z_{T}^{-1} Z_{g} g, \\
& f \rightarrow \stackrel{\circ}{f}=Z^{-1} Z_{T}^{-1} Z_{f} f,
\end{aligned}
$$

where the ${ }^{\circ}$ indicates unrenormalized quantities. Our scheme is chosen so that the Hamiltonian retains its orig- inal structure:

$$
\begin{aligned}
& \frac{\mathcal{H}}{T} \rightarrow \frac{1}{2 T} \int d^{d_{\perp}} x_{\perp} \int d x_{d}\left\{Z_{\omega} \omega u_{d d}^{2}+Z_{T}\left(\nabla_{\perp}^{2} u_{d}\right)^{2}\right. \\
& \left.+2 Z_{g} g u_{d d} u_{a a}+Z_{f} f u_{a a}^{2}+2 Z_{T} Z u_{a b} u_{a b}\right\}
\end{aligned}
$$

The simplest way of determining the renormalization Zfactors is minimal subtraction. In this procedure the Zfactors are chosen so that they solely cancel the $\varepsilon$ poles and otherwise leave the vertex functions unchanged. Expressed in terms of the effective couplings introduced in Sec. III our Z-factors are of the structure

$$
Z_{\ldots}(t, \sigma, \rho)=1+\sum_{m=1}^{\infty} \frac{X_{\ldots}^{(m)}(t, \sigma, \rho)}{\varepsilon^{m}} .
$$

The $X_{\ldots}^{(m)}(t, \sigma, \rho)$ are expansions in the effective temperature $t$ beginning with the power $t^{m}$. It is a fundamental fact of renormalization theory, cf. Ref. 23, that this procedure is suitable to eliminate all the UV-divergences (not only the superficial ones) from any vertex function order by order in perturbation theory. To one-loop order we find that our $\mathrm{Z}$ factors are given by

$$
\begin{aligned}
& Z=1+t \frac{14+13 \rho}{32 \pi \varepsilon \sqrt{2+\rho} \sqrt{2+\rho-\sigma}} \\
& Z_{T}=1-t \frac{3(1+\rho)}{8 \pi \varepsilon \sqrt{2+\rho} \sqrt{2+\rho-\sigma}} \\
& Z_{\omega}=1+t \frac{\sqrt{2+\rho} \sigma}{16 \pi \varepsilon \sqrt{2+\rho-\sigma}} \\
& Z_{g}=1+t \frac{\sqrt{2+\rho}(1+\rho)}{8 \pi \varepsilon \sqrt{2+\rho-\sigma}} \\
& Z_{f}=1+t \frac{\sqrt{2+\rho}\left(1+4 \rho+2 \rho^{2}\right)}{32 \pi \varepsilon \sqrt{2+\rho-\sigma}} .
\end{aligned}
$$

\section{Scaling 1: RG equation and its solution}

Next, we infer the scaling behavior of vertex function from a $\mathrm{RG}$ equation. This RG equation is a manifestation of the fact that the unrenormalized theory has to be independent of the arbitrary length scale $\mu^{-1}$ introduced by renormalization. By virtue of this independence, the unrenormalized vertex functions satisfy the identity

$$
\mu \frac{\partial}{\partial \mu} \stackrel{\circ}{\Gamma}^{(M, N)}\left(\left\{\mathbf{q}_{\perp}, q_{d}\right\} ; \stackrel{\circ}{\omega}, \stackrel{\circ}{T}, \stackrel{\circ}{g}, \stackrel{\circ}{f}\right)=0 .
$$


The identity (3.19) translates via the Wilson functions 31]

$$
\begin{aligned}
& \gamma_{\ldots}=\left.\mu \frac{\partial}{\partial \mu} \ln Z \ldots\right|_{0}, \\
& \zeta=\left.\mu \frac{\partial \ln \omega}{\partial \mu}\right|_{0}=\gamma_{T}-\gamma_{\omega}, \\
& \beta_{t}=\left.\mu \frac{\partial t}{\partial \mu}\right|_{0}=t\left(-\varepsilon-\gamma+\frac{1}{2} \gamma_{T}+\frac{1}{2} \gamma_{\omega}\right), \\
& \beta_{\sigma}=\left.\mu \frac{\partial \sigma}{\partial \mu}\right|_{0}=\sigma\left(\gamma+\gamma_{T}+\gamma_{\omega}-2 \gamma_{g}\right) \\
& \beta_{\rho}=\left.\mu \frac{\partial \rho}{\partial \mu}\right|_{0}=\rho\left(\gamma+\gamma_{T}-\gamma_{f}\right)
\end{aligned}
$$

into the Gell-Mann-Low RG equation

$$
\left[D_{\mu}-\left(M+\frac{N}{2}\right) \gamma\right] \Gamma^{(M, N)}\left(\left\{\mathbf{q}_{\perp}, q_{d}\right\} ; \omega, t, \sigma, \rho, \mu\right)=0 \text {. }
$$

Here we have used the shorthand notation

$$
D_{\mu}=\mu \frac{\partial}{\partial \mu}+\omega \zeta \frac{\partial}{\partial \omega}+\beta_{t} \frac{\partial}{\partial t}+\beta_{\sigma} \frac{\partial}{\partial \sigma}+\beta_{\rho} \frac{\partial}{\partial \rho} .
$$

The Wilson- $\gamma$-functions are easily gathered from the renormalization factors stated in Eqs. (3.18) upon reexpressing $\mu \frac{\partial}{\partial \mu}$ as $\beta_{t} \frac{\partial}{\partial t}$. Because the Wilson functions must be finite, one then immediately gets

$$
\gamma_{\ldots}(t, \sigma, \rho)=-t \partial_{t} X_{\ldots}^{(1)}(t, \sigma, \rho),
$$

where $X_{\ldots .}^{(1)}$ is defined in Eq. (3.17). Since we will need them to determine the fixed points of the RG flow, we state the Wilson- $\beta$-functions explicitly:

$$
\begin{aligned}
& \beta_{t}=-t \varepsilon+t^{2} \frac{20+19 \rho-2 \sigma-\rho \sigma}{32 \pi \sqrt{2+\rho} \sqrt{2+\rho-\sigma}}, \\
& \beta_{\sigma}=t \frac{\sqrt{2+\rho} \sigma(3+4 \rho-2 \sigma)}{32 \pi \sqrt{2+\rho-\sigma}} \\
& \beta_{\rho}=t \frac{2+7 \rho+7 \rho^{2}+2 \rho^{3}}{32 \pi \sqrt{2+\rho} \sqrt{2+\rho-\sigma}} .
\end{aligned}
$$

To solve the RG equation we employ the method of characteristics. We introduce a flow parameter $\ell$ and look for functions $\bar{\mu}(\ell), \bar{Z}(\ell), \bar{\omega}(\ell), \bar{t}(\ell), \bar{\sigma}(\ell)$, and $\bar{\rho}(\ell)$ determined by the characteristic equations

$$
\begin{aligned}
& \ell \frac{\partial \bar{\mu}(\ell)}{\partial \ell}=\bar{\mu}, \quad \bar{\mu}(1)=\mu, \\
& \ell \frac{\partial}{\partial \ell} \ln \bar{Z}(\ell)=\gamma(\bar{t}(\ell), \bar{\sigma}(\ell), \bar{\rho}(\ell)), \quad \bar{Z}(1)=1, \\
& \ell \frac{\partial}{\partial \ell} \ln \bar{\omega}(\ell)=\zeta(\bar{t}(\ell), \bar{\sigma}(\ell), \bar{\rho}(\ell)), \quad \bar{\omega}(1)=\omega, \\
& \ell \frac{\partial}{\partial \ell} \bar{t}(\ell)=\beta_{t}(\bar{t}(\ell), \bar{\sigma}(\ell), \bar{\rho}(\ell)), \quad \bar{t}(1)=t, \\
& \ell \frac{\partial}{\partial \ell} \bar{\sigma}(\ell)=\beta_{\sigma}(\bar{\sigma}(\ell), \bar{\sigma}(\ell), \bar{\rho}(\ell)), \quad \bar{\sigma}(1)=\sigma, \\
& \ell \frac{\partial}{\partial \ell} \bar{\rho}(\ell)=\beta_{\rho}(\bar{t}(\ell), \bar{\sigma}(\ell), \bar{\rho}(\ell)), \quad \bar{\rho}(1)=\rho .
\end{aligned}
$$

These characteristics describe how the parameters transform if we change the momentum scale $\mu$ according to $\mu \rightarrow \bar{\mu}(\ell)=\ell \mu$. Being interested in the IR behavior of the theory, we focus on the limit $\ell \rightarrow 0$. In this IR limit we find that the set of coupling constants $(\bar{t}(\ell), \bar{\sigma}(\ell), \bar{\rho}(\ell))$ flows to a stable fixed point

$$
\left(t^{*}, \sigma^{*}, \rho^{*}\right)=\left(\frac{32 \pi \varepsilon}{7}, 0,-\frac{1}{2}\right)
$$

satisfying $\beta_{t}\left(t^{*}, \sigma^{*}, \rho^{*}\right)=\beta_{\sigma}\left(t^{*}, \sigma^{*}, \rho^{*}\right)=$ $\beta_{\rho}\left(t^{*}, \sigma^{*}, \rho^{*}\right)=0$. Recalling that $\sigma=g^{2} / \omega$ and $\rho=f$, we learn that the stable fixed point implies two universal ratios of the elastic moduli (Poisson ratios):

$$
C_{2}^{2} /\left(C_{1} C_{4}\right)=0 \text { and } C_{3} / C_{4}=-1 / 2 .
$$

Note that the longitudinal and the transversal directions are effectively decoupled at the IR stable fixed point. In addition to the stable fixed point there are 4 unstable fixed points, viz. the zero temperature fixed point $t^{*}=0$ as well as $(32 \pi \varepsilon, 0,-1),(64 \sqrt{2 / 3} \pi \varepsilon / 13,1 / 2,-1 / 2)$, and $(32 \sqrt{2 / 3} \pi \varepsilon,-1 / 2,-1)$.

With help of the characteristics the RGE is readily solved, at least formally:

$$
\begin{aligned}
& \Gamma^{(M, N)}\left(\left\{\mathbf{q}_{\perp}, q_{d}\right\} ; \omega, t, \sigma, \rho, \mu\right)=\bar{Z}(\ell)^{-(M+N / 2)} \\
& \times \Gamma^{(M, N)}\left(\left\{\mathbf{q}_{\perp}, q_{d}\right\} ; \bar{\omega}(\ell), \bar{t}(\ell), \bar{\sigma}(\ell), \bar{\rho}(\ell), \mu \ell\right) .(3
\end{aligned}
$$

As it stands, Eq. (3.28) does not account for the naive dimensions of its ingredients. Recalling the $\mu$ invariance of the Hamiltonian $\mathcal{H}$ it is straightforward to check that

$$
\begin{aligned}
& \Gamma^{(M, N)}\left(\left\{\mathbf{q}_{\perp}, q_{d}\right\} ; \omega, t, \sigma, \rho, \mu\right) \\
& =\mu^{-(d+1)+d M+(d+1) N} \\
& \times \Gamma^{(M, N)}\left(\left\{\frac{\mathbf{q} \perp}{\mu}, \frac{q_{d}}{\mu^{2}}\right\} ; \omega, t, \sigma, \rho, 1\right) .
\end{aligned}
$$

Moreover, the $\beta$ invariance tells us that

$$
\begin{aligned}
& \Gamma^{(M, N)}\left(\left\{\mathbf{q}_{\perp}, q_{d}\right\} ; \omega, t, \sigma, \rho, \mu\right) \\
& =\beta^{(M+N-1)} \Gamma^{(M, N)}\left(\left\{\mathbf{q}_{\perp}, \frac{q_{d}}{\beta}\right\} ; \beta^{2} \omega, t, \sigma, \rho, \mu\right) \\
& =\omega^{-(M+N-1) / 2} \Gamma^{(M, N)}\left(\left\{\mathbf{q}_{\perp}, \omega^{1 / 2} q_{d}\right\} ; 1, t, \sigma, \rho, \mu\right) .
\end{aligned}
$$

where the last line reflects our freedom to chose $\beta=$ $\omega^{-1 / 2}$. Combining Eqs. (3.28), 3.29) we find that the scaling behavior of the vertex functions is described by

$$
\begin{aligned}
& \Gamma^{(M, N)}\left(\left\{\mathbf{q}_{\perp}, q_{d}\right\} ; \omega, t, \sigma, \rho, \mu\right) \\
& =(\mu \ell)^{-(d+1)+d M+(d+1) N} \bar{Z}(\ell)^{-(M+N / 2)} \\
& \times \Gamma^{(M, N)}\left(\left\{\frac{\mathbf{q}_{\perp}}{\mu \ell}, \frac{q_{d}}{(\mu \ell)^{2}}\right\} ; \bar{\omega}(\ell), \bar{t}(\ell), \bar{\sigma}(\ell), \bar{\rho}(\ell), \mu \ell\right) .
\end{aligned}
$$

Due to Eq. (3.30) we may also write

$$
\begin{aligned}
& \Gamma^{(M, N)}\left(\left\{\mathbf{q}_{\perp}, q_{d}\right\} ; \omega, t, \sigma, \rho, \mu\right) \\
& =(\mu \ell)^{-(d+1)+d M+(d+1) N} \bar{Z}(\ell)^{-(M+N / 2)} \bar{\omega}(\ell)^{-(M+N-1) / 2} \\
& \times \Gamma^{(M, N)}\left(\left\{\frac{\mathbf{q}_{\perp}}{\mu \ell}, \frac{\bar{\omega}(\ell)^{1 / 2} q_{d}}{(\mu \ell)^{2}}\right\} ; 1, \bar{t}(\ell), \bar{\sigma}(\ell), \bar{\rho}(\ell), \mu \ell\right) .
\end{aligned}
$$


We have to mention that Eqs. (3.31) and (3.32) do not correctly describe $\Gamma_{a b}^{(2,0)}$ at $\mathbf{q}_{\perp}=0$ because we omitted a dangerous irrelevant bending of the type $K_{d} \mathbf{q}_{d}^{4}$. Without such a term, Eqs. (3.31) and (3.32) unphysically suggest that the leading scaling behavior of $\Gamma_{a b}^{(2,0)}$ is independent of $q_{d}$ for $\mathbf{q}_{\perp}=0$. In other words, $K_{d}$ is dangerous irrelevant as far as the leading behavior of $\Gamma_{a b}^{(2,0)}$ at $\mathbf{q}_{\perp}=0$ is concerned. Because $K_{d}$ is irrelevant its omission has no impact on the leading behavior of the relevant elastic constants and hence does not affect our main results. Of course one could and it would be interesting to investigate the scaling behavior of $K_{d}$. One has to keep in mind, however, that irrelevant terms tend to mix under renormalization with a whole bunch of other irrelevant terms, making a proper RG analysis a tedious endevour. This will be left to future work.

\section{Scaling 2: Physical quantities}

The variables we considered so far in our RG analysis had the benefit of being convenient. The flip side of this convenience is, however, that the featured quantities have no direct physical meaning. Now we recast our results so that their physical content becomes pronounced.

To have a clear distinction between physical variables and the scaled variables we used in our calculations we mark the latter in the remainder of this section with a hat, i.e., we denote

$$
\hat{q}_{d}=\frac{C_{4}}{K^{2}} q_{d}, \hat{u}_{a}=\frac{C_{4}}{K} u_{a}, \hat{u}_{d}=\sqrt{\frac{C_{4}}{K}} u_{d} .
$$

It is not difficult to see that the relation between the physical vertex functions and the vertex functions in the scaled variables is given by

$$
\begin{aligned}
& \Gamma^{(M, N)}\left(\left\{\mathbf{q}_{\perp}, q_{d}\right\} ; \omega, t, \sigma, \rho, \mu\right) \\
& =K^{M+3 N / 2-2} C_{4}^{1-N / 2} \hat{\Gamma}^{(M, N)}\left(\left\{\mathbf{q}_{\perp}, \hat{q}_{d}\right\} ; \omega, t, \sigma, \rho, \mu\right) .
\end{aligned}
$$

Blending Eq. (3.34) and our findings of Sec. IIIB3 we now obtain

$$
\begin{aligned}
& \Gamma^{(M, N)}\left(\left\{\mathbf{q}_{\perp}, q_{d}\right\} ; \omega, t, \sigma, \rho, \mu\right)=\frac{1}{T} C_{1}^{-(M+N-2) / 2} \\
& \times C_{4}^{(2 M+N-2) / 2} K^{-(M-2) / 2} L_{\perp}^{2(d-1)-d M-(d+1) N} \\
& \times \ell^{-(d+1)+d M+(d+1) N}(\bar{\omega}(\ell) / \omega)^{-(M+N-1) / 2} \bar{Z}(\ell)^{-(M+N / 2)} \\
& \times \hat{\Phi}^{(M, N)}\left(\left\{\frac{L_{\perp} \mathbf{q}_{\perp}}{\ell}, \frac{\sqrt{\bar{\omega}(\ell) / \omega} L_{d} q_{d}}{\ell^{2}}\right\}\right. \\
& 1, \bar{t}(\ell), \bar{\sigma}(\ell), \bar{\rho}(\ell), 1)
\end{aligned}
$$

where we introduced the susceptibilities

$$
\hat{\Phi}^{(M, N)}=t \hat{\Gamma}^{(M, N)}
$$

to make explicit that the vertex functions are proportional to the inverse temperature. Moreover, we introduced the length scales

$$
\begin{aligned}
L_{\perp} & =\mu^{-1}, \\
L_{d} & =\frac{C_{4}}{K^{2}} \frac{\sqrt{\omega}}{\mu^{2}}=\sqrt{\frac{C_{1}}{K}} L_{\perp}^{2} .
\end{aligned}
$$

At this stage, $L_{\perp}$ and $L_{d}$ are still arbitrary. Further below we will fix these length scales so that they acquire a physical meaning, viz. the borderline between harmonic and scaling behavior. Note that the result (3.35) is general in the sense that it holds in the harmonic as well as in the scaling limit. When $\bar{t}(l) \approx 0$, the system behaves approximately like a harmonic system. When $l$ is small, behavior is determined by the fixed point with $t^{*} \sim \epsilon$.

Now to our main goal, viz. the behavior of the elastic constants. The sought after behavior can be inferred without much effort from result (3.35). As an example, we consider the case $M=0, N=2$ in some detail. In addition to the information contained in Eq. (3.35) we need some knowledge on the concrete form of the scaling function $\hat{\Phi}_{d d}^{(0,2)}$. Since $t^{*}$ is of order $\varepsilon$ it is reasonable to assume that $\hat{\Phi}_{d d}^{(0,2)}$ can be approximated by its Gaussian form [cf. Eq. [3.11)] even in the scaling limit. Hence, we write

$$
\begin{aligned}
& \hat{\Phi}_{d d}^{(0,2)}\left(\left\{\frac{L_{\perp} \mathbf{q}_{\perp}}{\ell}, \frac{\sqrt{\bar{\omega}(\ell) / \omega} L_{d} q_{d}}{\ell^{2}}\right\} ; 1, \bar{t}(\ell), \bar{\sigma}(\ell), \bar{\rho}(\ell), 1\right) \\
& =\left(\frac{\sqrt{\bar{\omega}(\ell) / \omega} L_{d} q_{d}}{\ell^{2}}\right)^{2}+\left(\frac{L_{\perp} \mathbf{q}_{\perp}}{\ell}\right)^{4} .
\end{aligned}
$$

Merging Eqs. (3.38) and (3.35) we obtain the physical vertex function

$$
\Gamma_{d d}^{(0,2)}\left(\mathbf{q}_{\perp}, q_{d}\right)=T^{-1}\left\{C_{1}(\ell) q_{d}^{2}+K(\ell)\left|\mathbf{q}_{\perp}\right|^{4}\right\}
$$

with

$$
\begin{aligned}
K(\ell) & =K \ell^{-\varepsilon} \bar{Z}(\ell)^{-1}[\bar{t}(\ell) / t]^{-1}[\bar{\omega}(\ell) / \omega]^{-1 / 2}, \\
C_{1}(\ell) & =C_{1} \ell^{-\varepsilon} \bar{Z}(\ell)^{-1}[\bar{t}(\ell) / t]^{-1}[\bar{\omega}(\ell) / \omega]^{1 / 2} .
\end{aligned}
$$

In the case of the bending modulus we cannot finalize our conclusions without solving the characteristics, i.e. without knowing $\bar{Z}(\ell)$ and so on. For $C_{1}$, however, we observe without further information the following:

$$
\ell \frac{\partial}{\partial \ell} C_{1}(\ell)=-\varepsilon-\gamma-\frac{\beta_{t}}{t}+\frac{\zeta}{2}=-\gamma_{\omega} .
$$

Since $\gamma_{\omega}$ is proportional to $\sigma$ it vanishes at the stable fixed point. Hence, $C_{1}$ is independent of $\ell$. In other words, $C_{1}$ is normal. Of course, we cannot tell from our analysis if this stays true beyond 1-loop order. Nevertheless, this may well be the case. 
The behavior of the remaining elastic constants can be extracted by similar means from the other 2-leg vertex functions. We find

$$
\begin{aligned}
\Gamma_{a d}^{(1,1)}\left(\mathbf{q}_{\perp}, q_{d}\right) & =T^{-1} C_{2}(\ell) q_{a} q_{d} \\
\Gamma_{a b}^{(2,0)}\left(\mathbf{q}_{\perp}, q_{d}\right) & =T^{-1}\left\{C_{3}(\ell) q_{a} q_{b}\right. \\
& \left.+C_{4}(\ell)\left(q_{a} q_{b}+\delta_{a b}\left|\mathbf{q}_{\perp}\right|^{2}\right)\right\}
\end{aligned}
$$

with the anomalous elastic constants

$$
\begin{aligned}
& C_{2}(\ell)=C_{2} \ell^{-\varepsilon} \bar{Z}(\ell)^{-3 / 2}[\bar{t}(\ell) / t]^{-1}[\bar{\sigma}(\ell) / \sigma]^{1 / 2}, \\
& C_{3}(\ell)=C_{3} \ell^{-\varepsilon} \bar{Z}(\ell)^{-2}[\bar{t}(\ell) / t]^{-1}[\bar{\omega}(\ell) / \omega]^{-1 / 2}, \\
& C_{4}(\ell) \sim C_{3}(\ell) .
\end{aligned}
$$

To obtain the equation for $C_{2}(\ell)$ we used the fact that it must be proportional to $C_{2}$ and thus to $\sigma^{1 / 2}$. Note that $\sigma$ affects the leading behavior of $C_{2}$ despite flowing to zero. In other words: $\sigma$ is a dangerous irrelevant variable. Similar arguments for $C_{3}$ imply that $C_{3}(\ell)$ must be proportional to $\rho$ which reaches a non-zero fixed point value. There are, therefore, no contributions to the scaling of $C_{3}$ from dangerous irrelevant variables.

\section{Behavior of the elastic constants in $d<3$}

For $\varepsilon>0$ we can assign a physical meaning to the hitherto arbitrary length scale $L_{\perp}$ via the definition of our dimensionless temperature $t$, viz.

$$
L_{\perp}=\left(\frac{\sqrt{C_{1} K^{3}} t}{C_{4} T}\right)^{1 / \varepsilon} .
$$

Of course, this choice also affects the length scale $L_{d}$, cf. Eq. 3.37b). The length scales $L_{\perp}$ and $L_{d}$ mark the borderline between harmonic and critical behavior.

Below 3 dimensions the solutions to the characteristics are for $\ell \ll 1$ governed by the IR-stable fixed point. Readily, one finds the power laws

$$
\begin{aligned}
& \bar{Z}(\ell)=\ell^{\gamma^{*}}, \\
& \bar{\omega}(\ell)=\omega \ell^{\zeta^{*}},
\end{aligned}
$$

where

$$
\begin{aligned}
& \gamma^{*}=\gamma\left(t^{*}, \sigma^{*}, \rho^{*}\right)=-5 \varepsilon / 7, \\
& \zeta^{*}=\zeta\left(t^{*}, \sigma^{*}, \rho^{*}\right)=4 \varepsilon / 7 .
\end{aligned}
$$

From our discussion above it is clear that we cannot simply set $\bar{\sigma}(\ell)$ equal to its fixed point value $\sigma^{*}=0$ because $\sigma$ is a dangerous irrelevant variable that effects the scaling behavior of $C_{2}$ at leading order. The vanishing of $\sigma$ is described by

$$
\bar{\sigma}(\ell) \sim \sigma \ell^{w},
$$

with a Wegner exponent $w=\varepsilon / 7$ corresponding to the smallest eigenvalue of the Hessian of the flow near $\left(t^{*}, \sigma^{*}, \rho^{*}\right)$.
Combining the formal scaling form of Eq. 3.35 with the power laws of Eq. (3.45) for $\bar{Z}(\ell), \bar{\omega}(\ell) / \omega$ and $\bar{\sigma}(\ell) / \sigma$ and the crossover length scales as given in Eqs. (3.44) and $(3.37 \mathrm{~b})$, we obtain a complete picture of the scaling behavior of the displacement vertex functions in $d<3$. In particular we obtain Eqs. (2.2) for the 2-point functions $\Gamma_{d d}^{(0,2)}, \Gamma_{a d}^{(1,1)}$ and $\Gamma_{a b}^{(2,0)}$ with $C_{5}=0$ featuring the scaling exponents

$$
\begin{aligned}
& \eta_{K}=\varepsilon+\gamma^{*}+\zeta^{*} / 2=4 \varepsilon / 7 \\
& \eta_{C}=-\varepsilon-2 \gamma^{*}-\zeta^{*} / 2=\varepsilon / 7 \\
& \eta_{2}=-\varepsilon-3 \gamma^{*} / 2+w / 2=\varepsilon / 7=\eta_{C} \\
& \phi=2-\zeta^{*} / 2=2-2 \varepsilon / 7=\left(4-\eta_{K}\right) / 2 .
\end{aligned}
$$

Thus, even though there are three independent exponents $\gamma^{*}, \zeta^{*}$ and $w$ the scaling behavior of the 2-point functions is determined, at least to first order in $\varepsilon$, by only two exponents, say $\eta_{K}$ and $\eta_{C}$. Upon inserting the power laws (3.45) into Eqs. (3.43) and (3.40a) we obtain the scaling expressions for $C_{2}, C_{3}, C_{4}$ and $K$ stated in Eqs. 2.5) and (2.6) the review section. Note that $K$ diverges at long length scales whereas $C_{2}, C_{3}$, and $C_{4}$ vanish in this limit.

\section{Logarithmic behavior in $d=3$}

Since $\varepsilon$ vanishes in $d=3$ the solutions to the characteristic equations are no longer of power law type. The flow of the temperature, for example, is described at leading order, i.e., for $\bar{\sigma}(\ell)=\sigma^{*}$ and $\bar{\rho}(\ell)=\rho^{*}$, by

$$
\ell \frac{\partial}{\partial \ell} \bar{t}(\ell)=\frac{7}{32 \pi} \bar{t}(\ell)^{2} .
$$

This differential equation is readily solved with the result

$$
\bar{t}(\ell) / t=\left[1-\frac{7 t}{32 \pi} \ln (\ell)\right]^{-1} .
$$

Similarly, we find $\bar{Z}(\ell) \sim[\bar{t}(\ell) / t]^{-\frac{5}{7}}, \bar{\omega}(\ell) \sim[\bar{t}(\ell) / t]^{\frac{4}{7}}$ and $\sigma(\ell) \sim[\bar{t}(\ell) / t]^{\frac{1}{7}}$ at leading order. Inserting these logarithmic solutions into Eqs. (3.40a) and (3.43) we find that

$$
\begin{aligned}
K\left(\mathbf{q}_{\perp}\right) & \sim K\left[1-\frac{7 t}{32 \pi} \ln \left(L_{\perp}\left|\mathbf{q}_{\perp}\right|\right)\right]^{4 / 7}, \\
C_{2}\left(\mathbf{q}_{\perp}\right) & \sim C_{2}\left[1-\frac{7 t}{32 \pi} \ln \left(L_{\perp}\left|\mathbf{q}_{\perp}\right|\right)\right]^{-1 / 7}, \\
C_{3}\left(\mathbf{q}_{\perp}\right) & \sim C_{4}\left(\mathbf{q}_{\perp}\right) \sim C_{2}\left(\mathbf{q}_{\perp}\right) .
\end{aligned}
$$

Of course, $C_{1}$ is normal as it was in $d<3$. Once more, $K$ diverges at long length scales and $C_{2}, C_{3}$, and $C_{4}$ vanish in this limit.

Note that Eqs. (3.49) imply the existence of a nonlinear crossover length scale, viz.

$$
\xi_{\perp}=L_{\perp} \exp \left(\frac{32 \pi}{7 t}\right)=L_{\perp} \exp \left(\frac{32 \pi \sqrt{C_{1} K^{3}}}{7 T C_{4}}\right) .
$$


For $\xi_{\perp}\left|\mathbf{q}_{\perp}\right| \approx 1$ the anomalous elastic constants are approximately harmonic whereas one has clearly anomalous behavior for $\xi_{\perp}\left|\mathbf{q}_{\perp}\right| \ll 1$.

\section{7. $C_{5}$ as a relevant perturbation - semi-soft elasticity}

Up to this point we have excluded a term proportional to $C_{5}$, cf. Eqs. (2.1) and (3.3), from the elastic energy of CSEs because such a term destroys the soft elasticity. Now we establish contact to more conventional uniaxial elastomers by incorporating a small but nonvanishing $C_{5}$. As a consequence, we find semi-soft behavior.

Technically, we treat $C_{5}$ as a perturbation to the CSE model. It turns out that this perturbation is relevant in the sense of the RG. This situation is analogous to the $\phi^{4}$ model where a deviation from the critical temperature represents a relevant perturbation. Our central task we will be to determine the scaling exponent that governs the departure of $C_{5}$ from zero.

Our analysis here is based on the full Hamiltonian (2.1). Carrying out the $\mu$-rescaling it is straightforward to see that not the entire strain $u_{a d}$ is relevant and that it is sufficient to keep

$$
u_{a d}=\frac{1}{2} \partial_{a} u_{d}
$$

Applying the rescaling that led us from Eq. (3.4) to (3.6) we obtain

$$
\mathcal{H}_{e}=\mathcal{H}+\frac{e}{2} \int d^{d_{\perp}} x_{\perp} \int d x_{d} \partial_{a} u_{d} \partial_{a} u_{d},
$$

with $\mathcal{H}$ as stated in Eq. (3.6) and where $e=C_{5} /(4 K)$. Note that $e \sim \mu^{2}$, i.e., the naive dimension of $e$ is 2 and hence $e$ is clearly relevant.

The Gaussian part of $\mathcal{H}_{e}$ has an extra term compared to $\mathcal{H}$ and hence the Gaussian propagator of $\mathcal{H}_{e}$ is different from that of $\mathcal{H}$. Here, any $\mathbf{q}_{\perp}^{4}$ in Eqs. (3.12) has to be replaced by $\mathbf{q}_{\perp}^{4}+e \mathbf{q}_{\perp}^{2}$. The non-Gaussian terms of $\mathcal{H}_{e}$ and $\mathcal{H}$ are identical and hence we still have the 4 vertices stated in Eqs. (3.13).

To investigate the departure of $e$ from zero we expand the propagator to linear order in $e$. Then this expanded propagator is used in our diagrammatic calculation. Of course, at zeroth order in $e$ we retrieve our vertex functions (3.14). The first order in the expansion leads to an extra divergent term in $\Gamma_{d d}^{(0,2)}$ that is proportional to $e \mathbf{q}_{\perp}^{2}$. To remove the extra divergence we introduce an additional renormalization factor via setting

$$
e \rightarrow \stackrel{\circ}{e}=Z_{T}^{-1} Z_{e} e .
$$

¿From the diagrams depicted in Fig. 11 we extract that

$$
Z_{e}=1-t \frac{6+5 \rho+\rho^{2}}{16 \pi \varepsilon \sqrt{2+\rho} \sqrt{2+\rho-\sigma}} .
$$

The RGE for the vertex functions expanded to linear order in $e$ reads

$$
\begin{aligned}
& {\left[D_{\mu}+e \psi \partial_{e}-\left(M+\frac{N}{2}\right) \gamma\right]} \\
& \times \Gamma^{(M, N)}\left(\left\{\mathbf{q}_{\perp}, q_{d}\right\} ; \omega, t, \sigma, \rho, e, \mu\right)=0
\end{aligned}
$$

with $D_{\mu}$ as stated in Eq. (3.22) and

$$
\psi=\left.\mu \partial_{\mu} \ln e\right|_{0}=\gamma_{T}-\gamma_{e}
$$

Setting up a characteristic for $e$,

$$
\ell \frac{\partial}{\partial \ell} \ln \bar{e}(\ell)=\psi(\bar{t}(\ell), \bar{\sigma}(\ell), \bar{\rho}(\ell)), \quad \bar{e}(1)=e,
$$

we find that this coupling flows in $d<3$ as

$$
\bar{e}(\ell) \sim e \ell^{\psi^{*}}
$$

where $\psi^{*}=\psi\left(t^{*}, \sigma^{*}, \rho^{*}\right)=-\varepsilon / 7$. Now, the solution to the RGE (3.55) in conjunction with dimensional analysis tells us that the scaling behavior of $\Gamma_{d d}^{(0,2)}$ expanded to linear order in $e$ is given by

$$
\begin{aligned}
& \Gamma_{d d}^{(0,2)}\left(\mathbf{q}_{\perp}, q_{d} ; \omega, t, \sigma, \rho, e, \mu\right)=(\mu \ell)^{4-\varepsilon} \bar{Z}(\ell)^{-1} \\
& \times \Gamma_{d d}^{(0,2)}\left(\frac{\mathbf{q}_{\perp}}{\mu \ell}, \frac{q_{d}}{(\mu \ell)^{2}} ; \bar{\omega}(\ell), \bar{t}(\ell), \bar{\sigma}(\ell), \bar{\rho}(\ell), \frac{\bar{e}(\ell)}{(\mu \ell)^{2}}, 1\right) .
\end{aligned}
$$

Next we switch back to physical variables. By performing much the same steps as in Secs. (IIIB 5) and (IIIB 4) we obtain for $d<3$

$$
\begin{aligned}
& \Gamma_{d d}^{(0,2)}\left(\mathbf{q}_{\perp}, q_{d} ; e\right)=\frac{K}{T} L_{\perp}^{-4} \ell^{4-\eta_{K}} \\
& \times \hat{\Phi}_{d d}^{(0,2)}\left(\frac{L_{\perp} \mathbf{q}_{\perp}}{\ell}, \frac{L_{d} q_{d}}{\ell^{\phi}} ; \frac{L_{\perp}^{2} e}{\ell^{1 / \nu_{5}}}\right),
\end{aligned}
$$

where we have dropped several arguments for notational simplicity and where

$$
\nu_{5}=\left(2-\psi^{*}\right)^{-1}=1 / 2-\varepsilon / 28 .
$$

Thus, $C_{5}$ plays the same role in this problem as temperature plays in a traditional thermal phase transition. As in the thermal case, it is useful to introduce a correlation length

$$
\xi_{5}=L_{\perp}\left(e L_{\perp}^{2}\right)^{-\nu_{5}} \sim C_{5}^{-\nu_{5}} .
$$

There are two interesting limits we can now consider: $\xi_{5}\left|\mathbf{q}_{\perp}\right| \ll 1$ and $\xi_{5}\left|\mathbf{q}_{\perp}\right| \gg 1$. In the first case, $\Gamma_{d d}^{(0,2)}$ must be proportional to $\mathbf{q}_{\perp}^{2}$ when $q_{d}=0$ and to $q_{d}^{2}$ when $\mathbf{q}_{\perp}=\mathbf{0}$. Thus, we obtain by choosing $\ell=\left(\xi_{5} / L_{\perp}\right)^{-\nu_{5}}$ from Eq. (3.60):

$$
\begin{aligned}
& \Gamma_{d d}^{(0,2)}\left(\mathbf{q}_{\perp}, q_{d} ; e\right) \\
& \sim \frac{1}{T}\left\{\begin{array}{lll}
K L_{\perp}^{-\eta_{K}} \xi_{5}^{-\left(2-\eta_{K}\right)} \mathbf{q}_{\perp}^{2} \sim C_{5}^{\gamma_{5}} \mathbf{q}_{\perp}^{2} & \text { if } & q_{d}=0 \\
C_{1} q_{d}^{2} & \text { if } & \mathbf{q}_{\perp}=\mathbf{0}
\end{array}\right.
\end{aligned}
$$


where

$$
\gamma_{5}=\nu_{5}\left(2-\eta_{K}\right)=1-5 \varepsilon / 14 .
$$

The second limit $\xi_{5}\left|\mathbf{q}_{\perp}\right| \gg 1$ corresponds $C_{5} \rightarrow 0$, and we must obtain the same scaling forms as we obtained for $C_{5}=0$ with a correction term that vanishes with $\left(\xi_{5}\left|\mathbf{q}_{\perp}\right|\right)^{-1 / \nu_{5}}$.

As we stated earlier, we have not included nonlinear terms in our model Hamiltonian [Eq. [3.4] ] that are needed to stabilize the system when $C_{5}<0$. In particular, we have not included terms $\left(u_{a d} u_{a d}\right)^{2} \sim\left(\partial_{a} u_{d} \partial_{a} u_{d}\right)^{2}$, $u_{a a} u_{b d} u_{b d}, \quad u_{a b} u_{a d} u_{b d}, \quad u_{a a}^{2} u_{b d} u_{b d}, \quad u_{a b}^{2} u_{c d} u_{c d}, \quad$ and $u_{a a} u_{b c} u_{b d} u_{c d}$, all of which have the same naive scaling dimension as the harmonic terms in nonlinear strain that we retained in Eq. (3.4). Our expectation is that the general theory in which these terms are included will have the same general form as the present theory with, however, a different stable fixed point with in particular a nonzero value of the coefficient of $\left(u_{a d} u_{a d}\right)^{2}$. At such a fixed point, $\left\langle\partial_{a} u_{d}\right\rangle$ will develop a nonzero value at negative $C_{5}$ that scales as $\left(-C_{5}\right)^{\beta}$ where $\beta=\left(1+\frac{1}{4} \gamma^{*}\right) \nu_{5}$. This result can be obtained by observing that Eq. (3.35) for $\Gamma^{(0,2)}$ implies $\partial_{a}^{2} G_{d d}\left(x_{d}, x_{a}, C_{5}\right)=$ $l^{2+\gamma^{*} / 2} \partial_{a}^{\prime 2} G_{d d}\left(l^{2+\zeta^{*} / 2} x_{d}, l x_{a}, l^{-\nu_{5}} C_{5}\right), \quad$ where $\partial_{a}^{\prime}$ is a derivative with respect to $l x_{a}$, and that $G_{d d}\left(x_{d}, x_{a}, C_{5}\right) \rightarrow\left\langle\partial_{a} u_{d}\right\rangle\left\langle\partial_{a} u_{d}\right\rangle$ as $\mathbf{x} \rightarrow \infty$.

\section{NEMATIC ELASTOMERS}

Usual NEs are either crosslinked in the isotropic or in the nematic phase. If synthesized in the isotropic phase, the uniaxial anisotropy arises via a spontaneous symmetry breaking at the isotropic to nematic transition and is associated with soft elasticity. Crosslinking in the nematic phase, on the other hand, permanently imprints the uniaxial anisotropy into the material and leads to semi-soft behavior. We will start by studying the soft case. Further below, we will include the effects of an imprinted uniaxial anisotropy to investigate the semi-soft case.

\section{A. The model}

Since the spontaneous symmetry axis of soft NEs can point in any direction, their elastic energy has to be rotationally invariant not only in target space but also in reference space. Both invariances are taken into account by writing the stretching energy as

$$
\begin{aligned}
& H_{\mathrm{st}}=\int d^{d} x\left\{\frac{\lambda}{2}(\operatorname{tr} \underline{\underline{u}})^{2}+\mu \operatorname{tr} \underline{\underline{u}}^{2}+A_{1}(\operatorname{tr} \underline{\underline{u}})^{3}\right. \\
& +A_{2} \operatorname{tr} \underline{\underline{u}} \operatorname{tr} \underline{\underline{u}}^{2}+A_{3} \operatorname{tr} \underline{\underline{u}}^{3}+B_{1}(\operatorname{tr} \underline{\underline{u}})^{4}+B_{2}\left(\operatorname{tr} \underline{\underline{u}}^{2}\right)^{2} \\
& \left.+B_{3}(\operatorname{tr} \underline{\underline{u}})^{2} \operatorname{tr} \underline{\underline{u}}^{2}+B_{4} \operatorname{tr} \underline{\underline{u}} \operatorname{tr} \underline{\underline{u}}^{3}+B_{5} \operatorname{tr} \underline{\underline{u}}^{4}\right\},
\end{aligned}
$$

with the first two expansion coefficients being the usual Lamé coefficients. Of course, terms of higher than fourth order are allowed by the symmetries of the system. However, these higher-order terms turn out to be irrelevant in the RG sense and are hence neglected.

Suppose that the spontaneously uniaxially ordered elastomer is described in equilibrium by an equilibrium strain tensor $\underline{\underline{u}}_{0}$. Without loss of generality we may assume that the anisotropy axis lies in the $\hat{\mathbf{e}}_{d}$ direction and that $\underline{\underline{u}}_{0}$ is a diagonal matrix with the diagonal elements $u_{0 a a}=u_{0 \perp}$ and $u_{0 d d}=u_{0 \|}$. To describe deviations from the equilibrium configuration, we introduce the relative strain

$$
\underline{\underline{w}}=\underline{\underline{u}}-\underline{\underline{u}}_{0}
$$

and to expand $\mathcal{H}$ in terms of $\underline{\underline{w}}$. By dropping terms that depend only on $\underline{\underline{u}}_{0}$ we find

$$
\begin{aligned}
& H_{\mathrm{st}}=\int d^{d_{\perp}} x_{\perp} \int d x_{d}\left\{a_{1} w_{d d}+a_{2} w_{a a}+b_{1} w_{d d}^{2}\right. \\
& +b_{2} w_{d d} w_{a a}+b_{3} w_{a a}^{2}+b_{4} w_{a b}^{2}+b_{5} w_{a d}^{2}+c_{1} w_{d d} w_{a d}^{2} \\
& \left.+c_{2} w_{a a} w_{b d}^{2}+c_{3} w_{a b} w_{a d} w_{b d}+d_{1} w_{a d}^{2} w_{b d}^{2}\right\}
\end{aligned}
$$

where we have discarded terms that turn out to be irrelevant. The new coefficients $a_{1}, a_{2}, b_{1}$, and so on, depend on the old coefficients $\lambda, \mu$, and so forth, as well as on $u_{0 \|}$ and $u_{0 \perp}$.

By virtue of the rotational invariance in reference space there exists a set Ward identities relating the vertex functions implicit in (4.3). We derive these identities in Appendix B At zero-loop or mean-field level these Ward identities correspond to relations between the elastic constants in (4.3),

$$
\begin{array}{ll}
a_{1}-a_{2}-s b_{5}=0, & \\
b_{2}-2 b_{3}-s c_{2}=0, & b_{2}+b_{5}-2 b_{1}+s c_{1}=0 \\
b_{5}-2 b_{4}-s c_{3}=0, & c_{1}-c_{2}-c_{3}-2 s d_{1}=0
\end{array}
$$

where $s$ is an abbreviation for $u_{0 \|}-u_{0 \perp}$. Since $\underline{\underline{w}}$ describes deviations from the equilibrium $\underline{\underline{u}}_{0}$ its thermal average $\langle\underline{\underline{w}}\rangle$ has to vanish. At zero temperature, where the mean-field approximation becomes exact, this means that the coefficients of the linear terms in (4.3) must be zero. Equation (4.4a) then leads to the observation that $b_{5}=0$ for $u_{0 \|} \neq u_{0 \perp}$. At finite temperatures thermal fluctuations become important and loop corrections renormalize the elastic constants including $a_{1}, a_{2}$ and $b_{5}$. For $\langle\underline{\underline{w}}\rangle$ to vanish the renormalized versions of $a_{1}$ and $a_{2}$ have to satisfy equations of state to which $a_{1}=0$ and $a_{2}=0$ are the mean-field approximations. In the following we will assume that we have chosen $a_{1}$ and $a_{2}$ appropriately so that their respective equations of state are satisfied. In other words: we assume that we expand about the true equilibrium state. Then, the Ward identity (B11) generalizing (4.4a) guarantees that the renormalized $b_{5}$ vanishes for $u_{0 \|} \neq u_{0 \perp}$. The vanishing of the elastic constant $b_{5}$ is the origin of the softness of NEs as can easily be seen by 
rewriting the nonvanishing terms of the Hamiltonian at leading order in small deformations in Fourier space.

In what follows we assume that $a_{1}=a_{2}=b_{5}=0$. Exploiting the relations (4.4) we rewrite the stretching energy as

$$
\begin{aligned}
H_{\mathrm{st}} & =\int d^{d_{\perp}} x_{\perp} \int d x_{d}\left\{b_{1} v_{d d}^{2}+b_{2} v_{d d} v_{a a}+b_{3} v_{a a}^{2}\right. \\
& \left.+b_{4} v_{a b}^{2}\right\}
\end{aligned}
$$

where we have introduced the non-standard strains

$$
\begin{aligned}
& v_{a b}=w_{a b}-s^{-1} w_{a d} w_{b d}, \\
& v_{d d}=w_{d d}+s^{-1} w_{a d} w_{a d} .
\end{aligned}
$$

Next we cast $v_{a b}$ and $v_{d d}$ into a more familiar form. Recall that the relative strain $\underline{w}$ depends upon the displacement $\mathbf{u}$ relative to the original isotropic state measured in the original reference space coordinate $\mathbf{x}$. It is more convenient, however, to work with a relative strain $\underline{u}^{\prime}$ that depends on the displacement $\mathbf{u}^{\prime}$ relative to the equilibrium state of the NE measured in the coordinate $\mathbf{x}^{\prime}$ of the corresponding uniaxial reference state. The tensors $\underline{\underline{w}}$ and $\underline{\underline{u}}^{\prime}$ are related via (see, e.g., Ref. [11])

$$
\underline{\underline{w}}=\underline{\underline{\Lambda}}_{0}^{T} \underline{\underline{u}}^{\prime} \underline{\underline{\Lambda}}_{0},
$$

where $\underline{\underline{\Lambda}}_{0}$ is the so-called Cauchy deformation tensor of the uniaxial equilibrium state. $\underline{\underline{\Lambda}}_{0}$ and $\underline{\underline{u}}_{0}$ provide equivalent descriptions of this state and they are related via

$$
\underline{\underline{u}}_{0}=\frac{1}{2}\left(\underline{\underline{\Lambda}}_{0}^{T} \underline{\underline{\Lambda}}_{0}-\underline{\underline{1}}\right) \text {, }
$$

where $\underline{\underline{1}}$ denotes the $d \times d$ unit matrix. Substituting the relation (4.7) into Eqs. (4.6) we obtain

$$
\begin{aligned}
& v_{a b}=\frac{\Lambda_{0 \perp}^{2}}{2}\left[\partial_{a}^{\prime} u_{b}^{\prime}+\partial_{b}^{\prime} u_{a}^{\prime}-\frac{1}{r-1} \partial_{a}^{\prime} u_{d}^{\prime} \partial_{a}^{\prime} u_{d}^{\prime}\right], \\
& v_{d d}=r \Lambda_{0 \perp}^{2}\left[\partial_{d}^{\prime} u_{d}^{\prime}+\frac{1}{2} \frac{1}{r-1} \partial_{a}^{\prime} u_{d}^{\prime} \partial_{a}^{\prime} u_{d}^{\prime}\right],
\end{aligned}
$$

where we have used Eqs. (3.5) and (3.51) to express $\underline{\underline{u}}^{\prime}$. $r=\Lambda_{0 \|}^{2} / \Lambda_{0 \perp}^{2}$ is the usual anisotropy ratio that characterizes the anisotropy of the uniaxial equilibrium state. In the steps leading to Eqs. (4.9) we have exploited that $s=\Lambda_{0 \perp}^{2}(r-1) / 2$. A glance at Eqs. (4.9) shows that we can write $\underline{\underline{v}}$ in a simpler and more traditional form by rescaling $\bar{x}_{a}^{\prime} \rightarrow x_{a}, x_{d}^{\prime} \rightarrow \sqrt{r-1} x_{d}, u_{a}^{\prime} \rightarrow u_{a}$ and $u_{d}^{\prime} \rightarrow \sqrt{r-1} u_{d}$. Incorporating bending, we eventually arrive at the Hamiltonian

$$
\begin{aligned}
\mathcal{H} & =\frac{1}{2} \int d^{d_{\perp}} x_{\perp} \int d x_{d}\left\{C_{1} v_{d d}^{2}+K\left(\nabla_{\perp}^{2} u_{d}\right)^{2}\right. \\
& \left.+2 C_{2} v_{d d} v_{a a}+C_{3} v_{a a}^{2}+2 C_{4} v_{a b}^{2}\right\}
\end{aligned}
$$

with the non-standard strains

$$
\begin{aligned}
v_{a b} & =\frac{1}{2}\left(\partial_{a} u_{b}+\partial_{b} u_{a}-\partial_{a} u_{d} \partial_{b} u_{d}\right), \\
v_{d d} & =\partial_{d} u_{d}+\frac{1}{2} \partial_{a} u_{d} \partial_{b} u_{d}
\end{aligned}
$$

Here, we exclusively retained the only bending term that is relevant in the $\mathrm{RG}$ sense. The new elastic constants $C_{\ldots}$ are proportional to the $b_{\ldots}$ in (4.5) $: C_{1}=2 b_{1} \Lambda_{0 \perp}^{4} r^{2} \sqrt{r-1}, C_{2}=b_{2} \Lambda_{0 \perp}^{4} r \sqrt{r-1}, C_{3}=$ $2 b_{3} \Lambda_{0 \perp}^{4} \sqrt{r-1}$ and $C_{4}=b_{4} \Lambda_{0 \perp}^{4} \sqrt{r-1}$.

A comment regarding the rescaling of $x_{d}$ is called for. Our rescalings $x_{d}^{\prime} \rightarrow \sqrt{r-1} x_{d}$ and $u_{d}^{\prime} \rightarrow \sqrt{r-1} u_{d}$ only make sense if $r>1$. We have assumed that the nematic phase is characterized by $r>1$. Nematic phases with $r<1$ are also possible. In this case, our rescalings would be different. At a second-order phase transition, $r-1$ will tend to zero. The transition from the isotropic to the nematic elastomer phase is first order in all dimensions above 2. The order of the transition in two dimensions has not yet been established. A second-order transition would have unusual properties since the coefficients of the nonlinear contributions to the nonlinear strain of Eqs. (4.9) diverge as $r \rightarrow 0$.

Instead of using the Hamiltonian (3.4) or our RG analysis we find it convenient to reduce the number of constants featured in the statistical weight $\exp (-\mathcal{H} / T)$ by rescaling $T \rightarrow \sqrt{K^{3} / C_{4}} T, x_{d} \rightarrow \sqrt{C_{4} / K} x_{d}, u_{d} \rightarrow$ $\sqrt{K / C_{4}} u_{d}$ and $u_{a} \rightarrow\left(K / C_{4}\right) u_{a}$. This gives us finally

$$
\begin{aligned}
\frac{\mathcal{H}}{T} & =\frac{1}{2 T} \int d^{d_{\perp}} x_{\perp} \int d x_{d}\left\{\omega v_{d d}^{2}+\left(\nabla_{\perp}^{2} u_{d}\right)^{2}\right. \\
& \left.+2 g v_{d d} v_{a a}+f v_{a a}^{2}+2 v_{a b}^{2}\right\}
\end{aligned}
$$

where

$$
\omega=C_{1} / C_{4}, \quad g=C_{2} / C_{4}, \quad f=C_{3} / C_{4} .
$$

Like the parametrization of Eq. (3.6) of the Hamiltonian for CSEs, the parametrization of Eq. (4.12) is not appropriate for taking the $C_{4} \rightarrow \infty$ limit to obtain a smectic- $A$ Hamiltonian. As was the case for CSEs, we are interested in properties unique to NEs, and we will not consider the Grinstein-Pelcovits limit of our model.

Formally, the Hamiltonians (3.6) and (4.12) look very similar. One has to bear in mind, however, that the strains $\underline{u}$ and $\underline{w}$ are different. In fact, the scaling symmetries of (3.6) and (4.12) are quite different except for the $\mu$ invariance. The naive dimensions of the fields, the temperature and the coupling constants are the same for the two models. In particular, NEs and CSEs have a mutual upper critical dimension $d_{c}=$ 3 . Though both systems possess of a mixing invariance the specific forms of these invariances are distinct: the NE Hamiltonian is invariant under the transformation $u_{a}\left(x_{c}, x_{d}\right) \rightarrow u_{a}\left(x_{c}-\theta_{c} x_{d}, x_{d}\right)+\theta_{a} u_{d}\left(x_{c}, x_{d}\right)$ and $u_{d}\left(x_{c}, x_{d}\right) \rightarrow u_{d}\left(x_{c}-\theta_{c} x_{d}, x_{d}\right)+\theta_{a} x_{a}$ provided that the $\theta$ 's are small. This mixing invariance is reminiscent of the original reference space rotation symmetry. In our current approach to NEs, the $\beta$-invariance of CSEs has counterpart. However, in Appendix D we present an alternative formulation that features a $\beta$-invariance at the cost of having an extra scaling parameter. Due to the different forms of the strains, the remaining symmetries stated at the end of Sec. ஹA have no analog in NEs. 


\section{B. Renormalization group analysis}

The diagrammatic perturbation expansions for CSEs and NEs are similar. Instead of repeating the details we will high-light the differences. In contrast, the RG behavior of CSEs and NEs is quite different due to the varying scaling symmetries.

\section{Diagrammatic expansion}

It is not difficult to see that the Gaussian propagator for NEs coincides with that for CSEs, see Eqs. (3.12). The differences between $\underline{\underline{u}}$ and $\underline{\underline{v}}$, however, lead to different vertices. For NEs we have

$$
\begin{aligned}
& i \frac{\omega-g}{2 T} q_{d}^{(1)} q_{b}^{(2)} q_{b}^{(3)} \\
& i \frac{g-f}{2 T} q_{a}^{(1)} q_{b}^{(2)} q_{b}^{(3)} \\
& i \frac{1}{T} q_{a}^{(2)} q_{b}^{(1)} q_{b}^{(3)} \\
& -\frac{\omega-2 g+f+2}{8 T} q_{a}^{(1)} q_{a}^{(2)} q_{b}^{(3)} q_{b}^{(4)}
\end{aligned}
$$

Of course the sum of the momenta has to vanish at each vertex. Note that the vertices (3.13) and (4.14) are of the same structure. Merely the coupling constants appear in different combination. Hence, the Feynman diagrams for both models have the same topology, or in other words, the 2-leg diagrams for both models can be drawn as in Figs. 11 to [3 Moreover, the same type of vertex functions are superficially divergent. By virtue of the NE mixing invariance, there exists a set of Ward identities relating the NE vertex functions. These identities are derived and stated in Appendix C. They guarantee that we merely have to calculate the 2-point functions. Appendix E describes details if the reader makes the appropriate changes in the vertices. Our results for the 2-point functions read

$$
\begin{aligned}
& \Gamma_{d d}^{(0,2)}=T^{-1}\left[\tau+\omega q_{d}^{2}+\mathbf{q}_{\perp}^{4}\right] \\
& -\frac{\sqrt{2+f}(g-\omega)^{2} q_{d}^{2}}{16 \pi \varepsilon \sqrt{-g^{2}+(2+f) \omega}} \tau^{-\varepsilon / 4} \\
& -\frac{\left[4 g+g^{2}-2(6+\omega)-f(12+\omega)\right] \mathbf{q}_{\perp}^{4}}{32 \pi \varepsilon \sqrt{2+f} \sqrt{-g^{2}+(2+f) \omega}} \tau^{-\varepsilon / 4} \\
& \Gamma_{a d}^{(1,1)}=T^{-1} g q_{a} q_{d} \\
& -\frac{\sqrt{2+f}(1+f-g)(g-\omega) q_{d} q_{a}}{16 \pi \varepsilon \sqrt{-g^{2}+(2+f) \omega}} \tau^{-\varepsilon / 4} \\
& \Gamma_{a b}^{(2,0)}=T^{-1}\left[(f+1) q_{a} q_{b}+\delta_{a b} \mathbf{q}_{\perp}^{2}\right] \\
& -\frac{\sqrt{2+f}\left[2(1+f-g)^{2} q_{a} q_{b}+\delta_{a b} \mathbf{q}_{\perp}^{2}\right]}{32 \pi \varepsilon \sqrt{-g^{2}+(2+f) \omega}} \tau^{-\varepsilon / 4} .
\end{aligned}
$$

\section{Renormalization}

We eliminate the $\varepsilon$ poles from the NE vertex functions by employing the renormalization scheme

$$
\begin{aligned}
& x_{d} \rightarrow \stackrel{\circ}{x}_{d}=Z^{-1 / 2} x_{d}, \\
& u_{d} \rightarrow \stackrel{\circ}{u}_{d}=Z^{1 / 2} u_{d}, \\
& u_{a} \rightarrow \stackrel{\circ}{u}_{a}=Z u_{a}, \\
& T \rightarrow \stackrel{\circ}{T}=Z^{1 / 2} Z_{T}^{-1} \mu^{\varepsilon} t, \\
& \omega \rightarrow \stackrel{\circ}{\omega}=Z^{-1} Z_{T}^{-1} Z_{\omega} \omega, \\
& g \rightarrow \stackrel{\circ}{g}=Z^{-1} Z_{T}^{-1} Z_{g} g, \\
& f \rightarrow \stackrel{\circ}{f}=Z^{-1} Z_{T}^{-1} Z_{f} f .
\end{aligned}
$$

Our renormalizations are devised so that the strains $v_{a b}$ and $v_{d d}$ as well as our Hamiltonian remain invariant in form,

$$
\begin{aligned}
& \frac{\mathcal{H}}{T} \rightarrow \frac{1}{2 T} \int d^{d_{\perp}} x_{\perp} \int d x_{d}\left\{Z_{\omega} \omega v_{d d}^{2}+Z_{T}\left(\nabla_{\perp}^{2} u_{d}\right)^{2}\right. \\
& \left.+2 Z_{g} g v_{d d} u_{a a}+Z_{f} f v_{a a}^{2}+2 Z_{T} Z v_{a b} v_{a b}\right\}
\end{aligned}
$$

The scheme 4.16) follows closely the approach developed by Grinstein and Pelcovitz 22]. Of course, other re-parameteriztions are conceivable. In Appendix [D we present an alternative formulation with a different renormalization scheme in which neither the elastic displacement nor $x_{d}$ is renormalized.

In our current formulation, there remanis no scaling invariance of the Hamiltonian that can be exploited to further reduce the number of coupling constants. Hence, the renormalization factors are functions of the original dimensionless parameters $t, \omega, g$ and $f$ rather than of a reduced number of effective couplings. The structure of the NE renormalization factors is

$$
Z_{\ldots}(t, \omega, g, f)=1+\sum_{m=1}^{\infty} \frac{X_{\ldots}^{(m)}(t, \omega, g, f)}{\varepsilon^{m}} .
$$

with $X_{\ldots}^{(m)}(t, \omega, g, f)$ being a power series in the effective temperature $t$ beginning with the power $t^{m}$. To 1-loop order we find from (4.15) via minimal subtraction

$$
\begin{aligned}
& Z=1+t \frac{4 g+g^{2}-2(7+\omega)-f(13+\omega)}{32 \pi \varepsilon \sqrt{2+f} \sqrt{-g^{2}+(2+f) \omega}}, \\
& Z_{T}=1-t \frac{4 g+g^{2}-2(6+\omega)-f(12+\omega)}{32 \pi \varepsilon \sqrt{2+f} \sqrt{-g^{2}+(2+f) \omega}}, \\
& Z_{\omega}=1+t \frac{\sqrt{2+f}(g-\omega)^{2}}{16 \pi \varepsilon \omega \sqrt{-g^{2}+(2+f) \omega}}, \\
& Z_{g}=1+t \frac{\sqrt{2+f}(1+f-g)(g-\omega)}{16 \pi \varepsilon g \sqrt{-g^{2}+(2+f) \omega}}, \\
& Z_{f}=1+t \frac{\sqrt{2+f}\left[1+4(f-g)+2(f-g)^{2}\right]}{32 \pi \varepsilon f \sqrt{-g^{2}+(2+f) \omega}} .
\end{aligned}
$$




\section{Scaling 1: RG equation and its solution}

The RGE for the NE vertex functions follows as ususal from the fact that the unrenormalized theory has to be independent of the arbitrary length scale $\mu^{-1}$ introduced by renormalization. Instead of working with the original parameters $\omega, g$ and $f$ we prefer to switch to

$$
\begin{aligned}
& \kappa=g / \omega=C_{2} / C_{1}, \quad \rho=f / \omega=C_{3} / C_{1}, \\
& \sigma=1 / \omega=C_{4} / C_{1} .
\end{aligned}
$$

This step turns out to be helpful in studying the RG flow because some of the original parameters tend to flow to infinity. We will see shortly that, on the other hand, $\kappa$, $\rho$ and $\sigma$ flow to finite values. Our RGE reads

$$
\left[D_{\mu}-\left(M+\frac{N}{2}\right) \gamma\right] \Gamma^{(M, N)}\left(\left\{\mathbf{q}_{\perp}, q_{d}\right\} ; t, \kappa, \rho, \sigma, \mu\right)=0
$$

with the RG differential operator

$$
D_{\mu}=\mu \partial_{\mu}-\frac{\gamma}{2} q_{d} \partial_{q_{d}}+\beta_{t} \partial_{t}+\beta_{\kappa} \partial_{\kappa}+\beta_{\rho} \partial_{\rho}+\beta_{\sigma} \partial_{\sigma} .
$$

The Wilson- $\beta$-functions, from which we determine the fixed points of the RG flow, are given in terms of the Wilson- $\gamma$-functions

$$
\gamma_{\ldots}=\left.\mu \frac{\partial}{\partial \mu} \ln Z_{\ldots}\right|_{0}
$$

by

$$
\begin{aligned}
& \beta_{t}=t\left(-\varepsilon+\gamma_{T}-\gamma / 2\right), \\
& \beta_{\kappa}=\kappa\left(\gamma_{\omega}-\gamma_{g}\right), \\
& \beta_{\rho}=\rho\left(\gamma_{\omega}-\gamma_{f}\right) \\
& \beta_{\sigma}=\sigma\left(\gamma_{\omega}-\gamma_{T}-\gamma\right) .
\end{aligned}
$$

The Wilson- $\gamma$-functions are readily extracted from the renormalization factors with the result

$$
\gamma_{\ldots}=-t \partial_{t} X_{\ldots}^{(1)}(t, \omega, g, f) \text {. }
$$

Switching to the parameters defined in Eqs. 4.20 we obtain

$$
\begin{aligned}
\beta_{t} & =-t \varepsilon \\
& -t^{2} \frac{3 \kappa^{2}+12 \kappa \sigma-2 \sigma(3+19 \sigma)-\rho(3+37 \sigma)}{64 \pi \sigma \sqrt{2+\rho / \sigma} \sqrt{\rho+2 \sigma-\kappa^{2}}} \\
\beta_{\kappa} & =t \frac{\sqrt{2+\rho / \sigma}(\kappa-1)\left(\rho+\sigma-\kappa^{2}\right)}{16 \pi \sqrt{\rho+2 \sigma-\kappa^{2}}} \\
\beta_{\rho} & =t \frac{\sqrt{2+\rho / \sigma}}{32 \pi \sqrt{\rho+2 \sigma-\kappa^{2}}} \\
& \times\left\{2 \kappa^{2}(1-\rho)+2 \rho^{2}-4 \kappa \sigma+\sigma^{2}+\rho(4 \sigma-2)\right\} \\
\beta_{\sigma} & =t \frac{\sqrt{2+\rho / \sigma} \sigma\left(-2+4 \kappa-2 \kappa^{2}+\sigma\right)}{32 \pi \sqrt{\rho+2 \sigma-\kappa^{2}}}
\end{aligned}
$$

For solving the RGE we introduce the characteristics

$$
\begin{aligned}
& \ell \frac{\partial \bar{\mu}(\ell)}{\partial \ell}=\bar{\mu}, \quad \bar{\mu}(1)=\mu \\
& \ell \frac{\partial}{\partial \ell} \ln \bar{Z}(\ell)=\gamma(\bar{t}(\ell), \bar{\kappa}(\ell), \bar{\sigma}(\ell), \bar{\rho}(\ell)), \quad \bar{Z}(1)=1 \\
& \ell \frac{\partial}{\partial \ell} \bar{t}(\ell)=\beta_{t}(\bar{t}(\ell), \bar{\kappa}(\ell), \bar{\sigma}(\ell), \bar{\rho}(\ell)), \quad \bar{t}(1)=t, \\
& \ell \frac{\partial}{\partial \ell} \bar{\kappa}(\ell)=\beta_{\kappa}(\bar{t}(\ell), \bar{\kappa}(\ell), \bar{\sigma}(\ell), \bar{\rho}(\ell)), \quad \bar{\kappa}(1)=\kappa, \\
& \ell \frac{\partial}{\partial \ell} \bar{\rho}(\ell)=\beta_{\rho}(\bar{t}(\ell), \bar{\kappa}(\ell), \bar{\sigma}(\ell), \bar{\rho}(\ell)), \quad \bar{\rho}(1)=\rho, \\
& \ell \frac{\partial}{\partial \ell} \bar{\sigma}(\ell)=\beta_{\sigma}(\bar{t}(\ell), \bar{\kappa}(\ell), \bar{\sigma}(\ell), \bar{\rho}(\ell)), \quad \bar{\sigma}(1)=\sigma
\end{aligned}
$$

In contrast to the CSE model we have to look for fixed points in a 4 dimensional parameter space. We find that the quadruple of coupling constants $(\bar{t}(\ell), \bar{\kappa}(\ell), \bar{\rho}(\ell), \bar{\sigma}(\ell))$ flows to the IR stable fixed point

$$
\left(t^{*}, \kappa^{*}, \rho^{*}, \sigma^{*}\right)=\left(\frac{64}{59} \sqrt{6} \pi \varepsilon, 1,1,0\right) .
$$

This fixed point is characterized by $C_{2} / C_{1}=\kappa^{*}=1$ and $C_{3} / C_{1}=\rho^{*}=1$. It turns out that the leading scaling behavior of physical quantities depends not only on the fixed point but also on the approach to the fixed point described by the dangerous irrelevant variable $\sigma$. Paths to the fixed point decay quickly to the line described by

$$
\bar{\kappa}(\ell)-\kappa^{*}=(a / 2+1 / 4) \bar{\sigma}(\ell)
$$

and

$$
\bar{\rho}(\ell)-\rho^{*}=a \bar{\sigma}(\ell)
$$

for small $\ell$, where $a$ is an arbitrary constant. In addition to the stable fixed point, there is the unstable Gaussian fixed point $t^{*}=0$ and there are two unstable fixed lines which can be parameterized as $(t(\sigma), \kappa(\sigma), \rho(\sigma), \sigma)$ with

$$
\begin{aligned}
t(\sigma) & =\frac{64 \pi \varepsilon \sqrt{2-2 \sqrt{2 \sigma}+3 \sigma}}{3136-3320 \sigma+1521 \sigma^{2}} \\
& \times\{56 \sqrt{2}+124 \sqrt{\sigma}+39 \sqrt{2} \sigma\}, \\
\kappa(\sigma) & =1-\sqrt{\sigma / 2} \\
\rho(\sigma) & =1-\sqrt{2 \sigma}-\sigma / 2
\end{aligned}
$$

and

$$
\begin{aligned}
t(\sigma) & =\frac{64 \pi \varepsilon \sqrt{2+2 \sqrt{2 \sigma}+3 \sigma}}{3136-3320 \sigma+1521 \sigma^{2}} \\
& \times\{56 \sqrt{2}-124 \sqrt{\sigma}+39 \sqrt{2} \sigma\}, \\
\kappa(\sigma) & =1+\sqrt{\sigma / 2} \\
\rho(\sigma) & =1+\sqrt{2 \sigma}-\sigma / 2 .
\end{aligned}
$$


With help of the characteristics a formal solution to the RGE is easily obtained,

$$
\begin{aligned}
& \Gamma^{(M, N)}\left(\left\{\mathbf{q}_{\perp}, q_{d}\right\} ; t, \kappa, \rho, \sigma, \mu\right)=\bar{Z}(\ell)^{-(M+1) / 2} \\
& \times \Gamma^{(M, N)}\left(\left\{\mathbf{q}_{\perp}, \bar{Z}(\ell)^{-1 / 2} q_{d}\right\} ; \bar{t}(\ell), \bar{\kappa}(\ell), \bar{\rho}(\ell), \bar{\sigma}(\ell), \mu \ell\right) .
\end{aligned}
$$

Dimensional analysis gives

$$
\begin{aligned}
& \Gamma^{(M, N)}\left(\left\{\mathbf{q}_{\perp}, q_{d}\right\} ; t, \kappa, \rho, \sigma, \mu\right)=\mu^{-(d+1)+d M+(d+1) N} \\
& \times \Gamma^{(M, N)}\left(\left\{\frac{\mathbf{q}_{\perp}}{\mu}, \frac{q_{d}}{\mu^{2}}\right\} ; t, \kappa, \rho, \sigma, 1\right) .
\end{aligned}
$$

Equation (4.32) in conjunction with (4.33) finally reveals the scaling behavior of the vertex functions:

$$
\begin{aligned}
& \Gamma^{(M, N)}\left(\left\{\mathbf{q}_{\perp}, q_{d}\right\} ; t, \kappa, \rho, \sigma, \mu\right) \\
& =(\mu \ell)^{-(d+1)+d M+(d+1) N} \bar{Z}(\ell)^{-(M+1) / 2} \\
& \times \Gamma^{(M, N)}\left(\left\{\frac{\mathbf{q}_{\perp}}{\mu \ell}, \frac{q_{d}}{(\mu \ell)^{2} \bar{Z}(\ell)^{1 / 2}}\right\} ;\right. \\
& \bar{t}(\ell), \bar{\kappa}(\ell), \bar{\rho}(\ell), \bar{\sigma}(\ell), \mu \ell) .
\end{aligned}
$$

\section{Scaling 2: physical quantities}

Now we switch back from the convenient scaled variables that we used in our calculations to the original variables in which we formulated our Hamiltonian (4.10). Once more, we mark rescaled variables as well as vertex functions of the rescaled variables with hats. Recalling our manipulations leading to Eq. (4.12) we write

$$
\begin{aligned}
& \hat{T}=\sqrt{\frac{C_{4}}{K^{3}}} T, \hat{t}=\sqrt{\frac{C_{4}}{K^{3}}} t, \\
& \hat{q}_{d}=\frac{C_{4}}{K} q_{d}, \hat{u}_{a}=\frac{C_{4}}{K} u_{a}, \hat{u}_{d}=\sqrt{\frac{C_{4}}{K}} u_{d} .
\end{aligned}
$$

The relation between the physical vertex functions and the vertex functions in the scaled variables is given by

$$
\begin{aligned}
& \Gamma^{(M, N)}\left(\left\{\mathbf{q}_{\perp}, q_{d}\right\} ; t, \kappa, \sigma, \rho, \mu\right) \\
& =K^{M+3 N / 2-7 / 2} C_{4}^{3 / 2-N / 2} \\
& \times \hat{\Gamma}^{(M, N)}\left(\left\{\mathbf{q}_{\perp}, \hat{q}_{d}\right\} ; \hat{t}, \kappa, \sigma, \rho, \mu\right) .
\end{aligned}
$$

Equation (4.36) in conjunction with Eq. (4.34) gives

$$
\begin{aligned}
& \Gamma^{(M, N)}\left(\left\{\mathbf{q}_{\perp}, q_{d}\right\} ; t, \kappa, \sigma, \rho, \mu\right) \\
& =\frac{1}{T} K^{M+3 N / 2-2} C_{4}^{1-N / 2} L_{\perp}^{2(d-1)-d M-(d+1) N} \\
& \times \ell^{-(d+1)+d M+(d+1) N}[\bar{t}(\ell) / t]^{-1} \bar{Z}(\ell)^{-(M+1) / 2} \\
& \times \hat{\Phi}^{(M, N)}\left(\left\{\frac{L_{\perp} \mathbf{q}_{\perp}}{\ell}, \frac{L_{d} q_{d}}{\bar{Z}(\ell)^{1 / 2} \ell^{2}}\right\} ;\right. \\
& \left.\quad \sqrt{\frac{C_{4}}{K^{3}}} \bar{t}(\ell), \bar{\kappa}(\ell), \bar{\sigma}(\ell), \bar{\rho}(\ell), 1\right),
\end{aligned}
$$

where we introduced the susceptibilities

$$
\hat{\Phi}^{(M, N)}=\hat{t} \hat{\Gamma}^{(M, N)}
$$

and where we switched from $\mu$ to the length scales

$$
\begin{aligned}
L_{\perp} & =\mu^{-1}, \\
L_{d} & =\sqrt{\frac{C_{4}}{K}} \mu^{-2}=\sqrt{\frac{C_{4}}{K}} L_{\perp}^{2} .
\end{aligned}
$$

The behavior of the elastic moduli is now easily extracted from the 2-point vertex functions. Upon specializing Eq. (4.37) to $N=2$ and $M=0$ etc. we obtain

$$
\begin{aligned}
K(\ell) & =K \ell^{-\varepsilon} \bar{Z}(\ell)^{-1 / 2}[\bar{t}(\ell) / t]^{-1}, \\
C_{1}(\ell) & =C_{1} \ell^{-\varepsilon} \bar{Z}(\ell)^{-3 / 2}[\bar{t}(\ell) / t]^{-1}[\bar{\sigma}(\ell) / \sigma]^{-1}, \\
C_{4}(\ell) & =C_{4} \ell^{-\varepsilon} \bar{Z}(\ell)^{-3 / 2}[\bar{t}(\ell) / t]^{-1} \\
C_{2}(\ell) & \sim C_{3}(\ell) \sim C_{1}(\ell)
\end{aligned}
$$

For $C_{1}, C_{2}$ and $C_{3}$ we can draw our final conclusions without solving the characteristics. Taking the derivative with respect to the flow parameter we find, for example,

$$
\ell \frac{\partial}{\partial \ell} C_{1}(\ell)=-\varepsilon-\frac{3}{2} \gamma-\frac{\beta_{t}}{t}-\frac{\beta_{\sigma}}{\sigma}=-\gamma_{\omega} .
$$

Upon expressing $\gamma_{\omega}$ in terms of $\sigma, \rho$, and $\kappa$ and by taking into account Eqs. (4.29) we see that

$$
\gamma_{\omega} \sim \sqrt{\sigma}
$$

in the vicinity of the IR-stable fixed point. Hence, $\gamma_{\omega}$ vanishes in the scaling limit. Consequently, $C_{1}, C_{2}$ and $C_{3}$ are normal.

\section{Behavior of the elastic constants for $d<3$}

For $d<3$ we can assign physical content to the length scale $L_{\perp}$ by exploiting the definition of the rescaled dimensionless temperature $\hat{t}$. This provides us with

$$
L_{\perp}=\left(\frac{K^{3 / 2} t}{C_{4}^{1 / 2} T}\right)^{1 / \varepsilon}
$$

as the transversal length scale associated with the onset of scaling behavior. The corresponding longitudinal length scale can be inferred from Eq. 4.39b.

As solutions to the characteristics we obtain the power laws

$$
\begin{aligned}
\bar{Z}(\ell) & \sim \ell^{\gamma^{*}}, \\
\bar{\sigma}(\ell) & \sim \sigma \ell^{w},
\end{aligned}
$$

where

$$
\gamma^{*}=\gamma\left(t^{*}, \kappa^{*}, \rho^{*}, \sigma^{*}\right)=-42 \varepsilon / 59
$$

and

$$
w=4 \varepsilon / 59
$$


is a Wegner exponent corresponding to the smallest eigenvalue of the Hessian at the stable fixed point.

Equation (4.37) in conjunction with the power-law behavior of $\bar{Z}(\ell)$ and $\bar{\sigma}(\ell) / \sigma$ along with Eqs. (4.43) and 4.39b for the crossover-lengths $L_{\perp}$ and $L_{d}$ provide us with a complete scaling picture of our vertex functions in $d<3$. This picture is summarized in Eqs. (2.13) with

$$
\begin{aligned}
& \eta_{K}=\varepsilon+\gamma^{*} / 2=38 \varepsilon / 59, \\
& \eta_{C}=\varepsilon+3 \gamma^{*} / 2=4 \varepsilon / 59=w, \\
& \phi=2+\gamma^{*} / 2=2-21 \varepsilon / 59 .
\end{aligned}
$$

Finally, the power-laws (4.44) along with Eqs. (4.40) result in the scaling forms for the elastic constants summarized in Eqs. (2.16) and (2.17). Note that K diverges at long length scales. $C_{4}$, on the other hand, vanishes in this regime.

\section{Logarithmic behavior in $d=3$}

In 3 dimensions the solutions to the characteristics that we need to determine the behavior of the elastic moduli are given at leading order by

$$
\bar{t}(\ell) / t=\left[1-\frac{7 \sqrt{6} t}{64 \pi} \ln (\ell)\right]^{-1}
$$

and $\bar{Z}(\ell) \sim[\bar{t}(\ell) / t]^{-\frac{42}{59}}$. Inserting these logarithmic solutions into Eqs. (4.40) we obtain

$$
\begin{aligned}
& K\left(\mathbf{q}_{\perp}\right) \sim K\left[1-\frac{7 \sqrt{6} t}{64 \pi} \ln \left(L_{\perp}\left|\mathbf{q}_{\perp}\right|\right)\right]^{38 / 59}, \\
& C_{4}\left(\mathbf{q}_{\perp}\right) \sim C_{4}\left[1-\frac{7 \sqrt{6} t}{64 \pi} \ln \left(L_{\perp}\left|\mathbf{q}_{\perp}\right|\right)\right]^{-4 / 59} .
\end{aligned}
$$

Using $\bar{\sigma}(\ell) \sim[\bar{t}(\ell) / t]^{\frac{4}{59}}$ one can check explicitly that $C_{1}$, $C_{2}$, and $C_{3}$ are normal. $K$ and $C_{4}$ diverge and vanish, respectively, for $L_{\perp}\left|\mathbf{q}_{\perp}\right| \rightarrow 0$. Note that Eqs. (4.47) imply the existence of the crossover-length scale

$$
\xi_{\perp}=L_{\perp} \exp \left(\frac{64 \pi}{7 \sqrt{6} t}\right)=L_{\perp} \exp \left(\frac{64 \pi \sqrt{K^{3}}}{7 \sqrt{6 C_{4}} T}\right) .
$$

\section{Poisson ratios}

Since the momentum dependence of the anomalous elastic moduli is logarithmic in 3 dimensions it will be difficult to observe the anomalous elasticity of NEs upon measuring these moduli directly. However, our analysis reveals the existence of several Poisson ratios of the elastic moduli that should be conveniently accessible by experiments.

The IR stable fixed point values of $\kappa, \rho$ and $\sigma$ directly imply the Poisson ratios $C_{2} / C_{1}=1, C_{3} / C_{1}=1$ and
$C_{4} / C_{1}=0$. Remarkably, not only the stable fixed point but also the approach to it contains information on relations between the elastic moduli. From Eqs. (4.29) it follows that $2 \kappa-\rho-1=\sigma / 2$ for small flow parameter $\ell$. Switching back to the original elastic constants we find the Poisson ratio

$$
\frac{2 C_{2}-C_{3}-C_{1}}{C_{4}}=\frac{1}{2} .
$$

Note that Eq. (4.49) implies the Poisson ratio $\mu / \mu_{L}=8$ found by Xing and Radzihovsky [24], where $\mu_{L}$ and $\mu$ are longitudinal and transverse shear moduli, respectively [32].

\section{Semi-soft elasticity}

So far we considered soft elastic NEs synthesized by crosslinking in the isotropic phase. If a NE is crosslinked in the nematic phase, a memory of the anisotropy at the time of crosslinking is locked in, or in other words, the rotational symmetry in the reference space is broken. The simplest way of modeling this symmetry breaking is by introducing an aligning external field, or more precisely, an aligning external stress. In this spirit we supplement the elastic energy (4.1) with the term stated in Eq. (2.11). Next, we switch to the relative strain (4.2) and exploit a Ward identity that corresponds at 0-loop order to

$$
h=2 s b_{5} .
$$

By virtue of this Ward identity, which is derived Appendix $B$ we can study the effects of the external aligning stress via studying the RG behavior of $b_{5}$ featured in Eq. (4.3).

Before embarking on a perturbation calculation we recall the rescalings that led from Eq. (4.3) to our final NE Hamiltonian (4.12). By applying these rescalings to Eq. (4.3) with now $b_{5}=h / s$ instead of $b_{5}=0$, by incorporating bending and by dropping irrelevant terms we derive the model Hamiltonian

$$
\frac{\mathcal{H}_{e}}{T}=\frac{\mathcal{H}}{T}+\frac{e}{2 T} \int d^{d_{\perp}} x_{\perp} \int d x_{d} \partial_{a} u_{d} \partial_{a} u_{d},
$$

with $\mathcal{H} / T$ given by Eq. (4.12). The coupling constant $e$ is defined as

$$
e=\frac{h}{4 K(1-2 s)} .
$$

Being interested in semi-soft behavior we assume that $h$ is small. Hence, we treat $e$ as a relevant perturbation.

Our diagrammatic calculation proceeds in much the same way as the one outlined in Sec.IIB 7 The only difference is that we here have to replace the vertices (3.13) by the NE vertices (4.14). Expansion of the diagrams to first order in $e$ leads to an $\varepsilon$ pole in $\Gamma_{d d}^{(0,2)}$ that is proportional to $e \mathbf{q}_{\perp}^{2}$. We remove the divergence by letting

$$
e \rightarrow \stackrel{\circ}{e}=Z_{T}^{-1} Z_{e} e
$$


with

$$
Z_{e}=1-t \frac{\sqrt{2+f}(3+f-2 g+\omega)}{16 \pi \varepsilon \sqrt{-g^{2}+(2+f) \omega}} .
$$

The appropriate RGE here reads

$$
\begin{aligned}
& {\left[D_{\mu}+e \psi \partial_{e}-\left(M+\frac{N}{2}\right) \gamma\right]} \\
& \times \Gamma^{(M, N)}\left(\left\{\mathbf{q}_{\perp}, q_{d}\right\} ; t, \kappa, \rho, \sigma, e, \mu\right)=0
\end{aligned}
$$

where it is understood the vertex functions are expanded to first order in $e$. The RG differential operator is $D_{\mu}$ is stated in Eq. (4.22) and

$$
\psi=\left.\mu \partial_{\mu} \ln e\right|_{0}=\gamma_{T}-\gamma_{e} .
$$

In $d<3$ the characteristic for $e$,

$$
\ell \frac{\partial}{\partial \ell} \ln \bar{e}(\ell)=\psi(\bar{t}(\ell), \bar{\kappa}(\ell), \bar{\rho}(\ell), \bar{\sigma}(\ell)), \bar{e}(1)=e,
$$

has the fixed point solution

$$
\bar{e}(\ell) \sim e \ell^{\psi^{*}},
$$

with $\psi^{*}=\psi\left(t^{*}, \kappa^{*}, \rho^{*}, \sigma^{*}\right)=18 \varepsilon / 59$. Supplementing the solution to the RGE (4.55) with a dimensional analysis and switching to original variables yields for the physical vertex function $\Gamma_{d d}^{(0,2)}$ the scaling form

$$
\begin{aligned}
& \Gamma_{d d}^{(0,2)}\left(\mathbf{q}_{\perp}, q_{d} ; e\right)=\frac{K}{T} L_{\perp}^{-4} \ell^{4-\eta_{K}} \\
& \times \hat{\Phi}_{d d}^{(0,2)}\left(\frac{L_{\perp} \mathbf{q}_{\perp}}{\ell}, \frac{L_{d} q_{d}}{\ell^{\phi}} ; \frac{L_{\perp}^{2} e}{\ell^{1 / \nu_{h}}}\right),
\end{aligned}
$$

where we have simplified the notation by dropping several arguments and where

$$
\nu_{h}=\left(2-\psi^{*}\right)^{-1}=1 / 2+9 \varepsilon / 108 .
$$

We emphasize the different roles played by $C_{5}$ and $h$ in the CSE model and in NEs, respectively. $C_{5}$ corresponds to a temperature whereas $h$ corresponds to an external magnetic field. Nevertheless, it is useful to introduce a length scale

$$
\xi_{h}=L_{\perp}\left(e L_{\perp}^{2}\right)^{-\nu_{h}} \sim h^{-\nu_{h}}
$$

at this point. In the limit $\xi_{h}\left|\mathbf{q}_{\perp}\right| \ll 1$ we obtain upon choosing $\ell=\left(\xi_{h} / L_{\perp}\right)^{-\nu_{h}}$ that

$$
\begin{aligned}
& \Gamma_{d d}^{(0,2)}\left(\mathbf{q}_{\perp}, q_{d} ; e\right) \\
& \sim \frac{1}{T}\left\{\begin{array}{lll}
K L_{\perp}^{-\eta_{K}} \xi_{h}^{-\left(2-\eta_{K}\right)} \mathbf{q}_{\perp}^{2} \sim h^{\gamma_{h}} \mathbf{q}_{\perp}^{2} & \text { if } & q_{d}=0 \\
C_{1} q_{d}^{2} & \text { if } & \mathbf{q}_{\perp}=\mathbf{0}
\end{array}\right.
\end{aligned}
$$

where

$$
\gamma_{h}=\nu_{h}\left(2-\eta_{K}\right)=1-10 \varepsilon / 59 .
$$

In the limit $\xi_{h}\left|\mathbf{q}_{\perp}\right| \gg 1$, corresponding to the limit $h \rightarrow 0$, we retrieve essentially the scaling form that we had for $h=0$. Now, however, there is an additional correction term that vanishes as $\left(\xi_{h}\left|\mathbf{q}_{\perp}\right|\right)^{-1 / \nu_{h}}$. Physically, this correction term modifies the behavior from soft to semi-soft. For a review of the complete scaling results, see Eqs. (2.13), (2.16) and (2.17).

\section{CONCLUDING REMARKS}

In this paper, we have explored the anomalous elasticity of two models for soft uniaxial elastomers, both of which are characterized by the vanishing of the shear modulus $C_{5}$ for shears in planes containing the anisotropy axis. The first model, which we refer to as a critically soft elastomer (CSE), describes a uniaxial system at a simplified critical point separating a true uniaxial elastic phase characterized by five elastic moduli and a lower symmetry phase produced by shearing the uniaxial solid. The second model describes nematic elastomers (NEs) formed via spontaneous symmetry breaking from an anisotropic state. In the CSE model, a bending modulus diverges, and three of the four elastic moduli vanish as power-laws in wavenumber at long wavelength below spatial dimension $d=3$ and logarithmically at $d=3$. In the NE model, the relevant bending modulus diverges, but only one elastic constant exhibits singular behavior. In both models, we studied the effect of turning on couplings that take them from soft to true uniaxial elastomers. These fields act like temperature or external fields at a thermal critical point, respectively, and introduce coherence lengths that diverge as a power law as the fully soft state is approached.

The logarithmic corrections that we predict will be very difficult to measure. Our universal Poisson ratios, on the other hand, should be observable in experiments on three-dimensional soft elastomers. The anomalous elasticity will be more important in 2 than in 3 dimensions. It would be interesting to find a two-dimensional realization of nematic elastomers, say in a crosslinked membrane confined to a substrate which inhibits height fluctuations 33. Another two-dimensional system belonging the NE universality class would be a tethered nematic membrane that orients in a plane perpendicular to an external aligning field.

Our analysis exemplifies the power and the beauty of the renormalization group. The renormalization group handles the rotational invariances of nematic elastomers in two distinct spaces quite naturally. Though the constraints imposed by these invariances lead to almost baroquely complicated formulae at intermediate stages of the analysis, our final results have a surprisingly clear and simple stature.

\section{Acknowledgments}

We gratefully acknowledge financial support by the Emmy Noether-Programm of the Deutsche Forschungsgemeinschaft (OS) and the National Science Foundation (TCL, grant DMR 00-96531). We thank H. K. Janssen and J. Toner for fruitful discussions. We are particularly grateful for discussions with X. Xing, and L. Radzihovsky, who have also studied this problem. 


\section{APPENDIX A: WARD IDENTITIES FOR CRITICALLY SOFT ELASTOMERS}

By virtue of the mixing invariance discussed a the end of Sec. IIIA there exist several Ward identities for CSEs. In this Appendix we derive these identities.

To facilitate our derivation, we introduce external fields via

$$
\mathcal{H} \rightarrow \mathcal{H}_{h}=\mathcal{H}-\int d^{d} x\left\{h_{a} u_{a}+h_{d} u_{d}\right\},
$$

where $\int d^{d} x=\int d^{d_{\perp}} x_{\perp} \int d x_{d}$. As usual, the external fields allow us to exploit the free energy

$$
F\left[h_{a}, h_{d}\right]=-T \ln Z\left[h_{a}, h_{d}\right],
$$

where the partition function is given by

$$
Z\left[h_{a}, h_{d}\right]=\int \mathcal{D} u_{a} \mathcal{D} u_{d} \exp \left(-\mathcal{H}_{h} / T\right),
$$

as a generating function for the order parameters

$$
\begin{aligned}
& m_{a}(\mathbf{x})=\left\langle u_{a}(\mathbf{x})\right\rangle=\frac{\delta F}{\delta h_{a}(\mathbf{x})}, \\
& m_{d}(\mathbf{x})=\left\langle u_{d}(\mathbf{x})\right\rangle=\frac{\delta F}{\delta h_{d}(\mathbf{x})} .
\end{aligned}
$$

As a consequence of the mixing invariance, the free energy obeys the relation

$$
F\left[h_{a}, h_{d}\right]=\theta_{a} \int d^{d} x x_{a} h_{d}+F\left[h_{a}, h_{d}^{\prime}\right],
$$

where $h_{d}^{\prime}=h_{d}+\theta_{a} h_{a}$. Because the right hand side of Eq. (A6) is independent of the $\theta_{a}$, we obtain

$$
\begin{aligned}
& L_{a}=\frac{\partial F}{\partial \theta_{a}}=\int d^{d} x x_{a} h_{d}(\mathbf{x})+\int d^{d} x m_{d}(\mathbf{x}) h_{a}(\mathbf{x}) \\
& =0 .
\end{aligned}
$$

Now we adopt the usual strategy and take various derivatives. We start with

$$
\begin{aligned}
& \frac{\delta^{2} L_{a}}{\delta m_{d}(\mathbf{y}) \delta m_{b}(\mathbf{z})}=\int d^{d} x x_{a} \frac{\delta^{2} h_{d}(\mathbf{x})}{\delta m_{d}(\mathbf{y}) \delta m_{b}(\mathbf{z})} \\
& +\frac{\delta h_{a}(\mathbf{y})}{\delta m_{b}(\mathbf{z})}+\int d^{d} x m_{d}(\mathbf{x}) \frac{\delta^{2} h_{a}(\mathbf{x})}{\delta m_{d}(\mathbf{y}) \delta m_{b}(\mathbf{z})}=0
\end{aligned}
$$

In equilibrium, the order parameters vanish for vanishing external fields, $h_{a}=h_{d}=0$. Hence, we get the Ward identity

$$
-\int d^{d} x x_{a} \Gamma_{d d b}^{(1,2)}(\mathbf{x}, \mathbf{y}, \mathbf{z})=\Gamma_{a b}^{(2,0)}(\mathbf{y}, \mathbf{z}) .
$$

In Fourier space, identity (A9) takes the form

$$
-\left.i \frac{\partial}{\partial p_{a}^{(1)}} \Gamma_{d d b}^{(1,2)}\left(\mathbf{p}^{(1)}, \mathbf{p}^{(2)}, \mathbf{p}^{(3)}\right)\right|_{\mathbf{p}^{(1)}=\mathbf{0}}=\Gamma_{a b}^{(2,0)}\left(\mathbf{p}^{(2)}, \mathbf{p}^{(3)}\right)
$$

with $\mathbf{p}^{(2)}+\mathbf{p}^{(3)}=\mathbf{0}$. Another Ward identity can be found by taking the second functional derivative of $L_{a}$ with respect to $m_{d}(\mathbf{y})$ and $m_{d}(\mathbf{z})$. This leads in Fourier space to

$$
\begin{aligned}
& -\left.i \frac{\partial}{\partial p_{a}^{(1)}} \Gamma_{d d d}^{(0,3)}\left(\mathbf{p}^{(1)}, \mathbf{p}^{(2)}, \mathbf{p}^{(3)}\right)\right|_{\mathbf{p}^{(1)}=\mathbf{0}} \\
& =\Gamma_{a d}^{(1,1)}\left(\mathbf{p}^{(2)}, \mathbf{p}^{(3)}\right)+\Gamma_{a d}^{(1,1)}\left(\mathbf{p}^{(3)}, \mathbf{p}^{(2)}\right)
\end{aligned}
$$

An identity for the four point vertex function $\Gamma_{d d d d}^{(0,4)}$ follows in a similar manner upon taking a third order functional derivative of $L_{a}$ with respect to, say, $m_{d}(\mathbf{y}), m_{d}(\mathbf{z})$, and $m_{d}(\mathbf{w})$. The result can be stated as

$$
\begin{aligned}
& -\left.i \frac{\partial}{\partial p_{a}^{(1)}} \Gamma_{d d d d}^{(0,4)}\left(\mathbf{p}^{(1)}, \mathbf{p}^{(2)}, \mathbf{p}^{(3)}, \mathbf{p}^{(4)}\right)\right|_{\mathbf{p}^{(1)}=\mathbf{0}} \\
& =\Gamma_{\text {add }}^{(1,2)}\left(\mathbf{p}^{(2)}, \mathbf{p}^{(3)}, \mathbf{p}^{(4)}\right)+\Gamma_{\text {add }}^{(1,2)}\left(\mathbf{p}^{(4)}, \mathbf{p}^{(2)}, \mathbf{p}^{(3)}\right) \\
& +\Gamma_{\text {add }}^{(1,2)}\left(\mathbf{p}^{(3)}, \mathbf{p}^{(4)}, \mathbf{p}^{(2)}\right) .
\end{aligned}
$$

Of course, our Ward identities should hold to arbitrary order in perturbation theory. At zero loop order, the consistency of the Ward identities can be checked without much effort. We carried out this check and found our identities confirmed.

\section{APPENDIX B: WARD IDENTITIES FOR NEMATIC ELASTOMERS I}

Here we derive the Ward identities that follow from the reference space rotation invariance of the NE Hamiltonian (4.1). One of these Ward identities can be viewed as the origin of the soft elasticity characterizing NEs crosslinked in the isotropic phase. In addition, we consider semi-soft NEs where the reference space rotation invariance is broken by an aligning external stress.

\section{The soft case}

To make our arguments more intuitive we work directly in $d=3$ dimensions. Without loss of generality we assume rotations about the $x$ axis which we parameterize by a rotation angle $\theta$. For small $\theta$ these rotations are described by the orthogonal matrix

$$
\underline{\underline{O}}_{R}=\left(\begin{array}{ccc}
1 & 0 & 0 \\
0 & 1 & \theta \\
0 & -\theta & 1
\end{array}\right) .
$$

Such a rotation takes the strain $\underline{\underline{u}}$ to $\underline{\underline{u}}^{\prime}=\underline{\underline{O}} R \underline{\underline{u}} \underline{\underline{O}}_{R}^{T}$, where $\underline{\underline{O}}_{R}^{T}$ is the transposed of $\underline{\underline{O}}_{R}$. The relative strain $\underline{\underline{w}}=$ $\underline{\underline{u}}-\underline{\underline{u}}_{0}$ is taken by the rotation to

$$
\underline{\underline{w}}^{\prime}=\underline{\underline{O}}_{R} \underline{\underline{u}}_{0} \underline{\underline{O}}_{R}^{T}-\underline{\underline{u}}_{0}+\underline{\underline{O}}_{R} \underline{\underline{w}}_{\underline{O}}^{T} .
$$


We introduce an external symmetric stress $\underline{\underline{\sigma}}$ into our model via

$$
\mathcal{H} \rightarrow \mathcal{H}_{\sigma}=\mathcal{H}-\int d^{d} x \sigma_{i j} w_{i j}
$$

where $\mathcal{H}$ is the elastic energy of Eq. (4.3) supplemented with the relevant bending term. Then, the free energy

$$
F[\underline{\underline{\sigma}}]=-T \ln Z[\underline{\underline{\sigma}}],
$$

with the partition function

$$
Z[\underline{\underline{\sigma}}]=\int \mathcal{D} \underline{\underline{w}} \exp \left(-\mathcal{H}_{\sigma} / T\right)
$$

is a generating function for the tensor order parameter

$$
m_{i j}(\mathbf{x})=\left\langle w_{i j}(\mathbf{x})\right\rangle=\frac{\delta F}{\delta \sigma_{i j}(\mathbf{x})} .
$$

Owing to the rotation invariance of the Hamiltonian (4.3) without external stress the free energy satisfies

$$
F[\underline{\underline{\sigma}}]=-\theta s \int d^{d} x\left[\sigma_{23}+\sigma_{32}\right]+F\left[\underline{\underline{\sigma}}^{\prime}\right],
$$

with the elements of the symmetric tensor $\underline{\underline{\sigma^{\prime}}}$ given by

$$
\begin{aligned}
\sigma_{11}^{\prime} & =\sigma_{11} \\
\sigma_{12}^{\prime} & =\sigma_{12}-\theta \sigma_{13} \\
\sigma_{13}^{\prime} & =\sigma_{13}+\theta \sigma_{12}, \\
\sigma_{22}^{\prime} & =\sigma_{22}-\theta\left(\sigma_{23}+\sigma_{32}\right), \\
\sigma_{23}^{\prime} & =\sigma_{23}+\theta\left(\sigma_{22}-\sigma_{33}\right), \\
\sigma_{33}^{\prime} & =\sigma_{33}+\theta\left(\sigma_{23}+\sigma_{32}\right) .
\end{aligned}
$$

Evidently, the left hand side of the identity (B7) is independent of the rotation angle $\theta$. Hence, the quantity

$$
\begin{aligned}
L & =\frac{\partial F}{\partial \theta}=\int d^{d} x\left\{-s \Gamma_{23}(\mathbf{x})+m_{12} \Gamma_{13}(\mathbf{x})\right. \\
& -m_{13} \Gamma_{12}(\mathbf{x})-2 m_{23}\left[\Gamma_{22}(\mathbf{x})-\Gamma_{33}(\mathbf{x})\right] \\
& \left.-\left[m_{33}(\mathbf{x})-m_{22}(\mathbf{x})\right] \Gamma_{23}(\mathbf{x})\right\}
\end{aligned}
$$

vanishes identically. In this expression, we used the facts that $m_{i j}(\mathbf{x})$ and $\sigma_{i j}$ are symmetric tensors and that $\Gamma_{i j}(\mathbf{x})=2 \delta F / \delta m_{i j}(\mathbf{x})$ for $i \neq j$ (we use only $i<j$ ) and $\Gamma_{i i}(\mathbf{x})=\delta F / \delta m_{i i}(\mathbf{x})$ for all $i$ (no Einstein convention). Now we are in the position to extract the sought after Ward identities simply by taking functional derivatives of $L$ with respect to the order parameter. For example, differentiating with respect to $m_{23}(\mathbf{y})$ and setting $m_{i j}=0$, we obtain the Ward identity,

$$
\begin{aligned}
& \frac{\delta L}{\delta m_{23}(\mathbf{y})}= \\
& -s \int d^{d} x \Gamma_{2323}(\mathbf{x}, \mathbf{y})-2\left[\Gamma_{22}(\mathbf{y})-\Gamma_{33}(\mathbf{y})\right]=0
\end{aligned}
$$

which in Fourier space is

$$
s \Gamma_{2323}(\mathbf{0}, \mathbf{0})+2\left[\Gamma_{22}(\mathbf{0})-\Gamma_{33}(\mathbf{0})\right]=0 .
$$

This Ward identity is particularly important because it is the origin of the soft elasticity of NEs. Taking the appropriate derivatives of the free energy in Eq. (4.3), it is straightforward to show that $\Gamma_{2323}(\mathbf{0}, \mathbf{0})=2 b_{5}$, $\Gamma_{33}(\mathbf{0}, \mathbf{0})=a_{1}$, and $\Gamma_{22}(\mathbf{0}, \mathbf{0})=a_{2}$ and thus that Eq. (B11) reduces relation (4.4a) at 0 -loop order.

The derivation of the other Ward identities that generalize the remaining relations stated in Eqs. (4.4) is a similar exercise. We restrict ourselves to stating the final results

$$
\begin{aligned}
& -s \Gamma_{232311}(\mathbf{0},-\mathbf{p}, \mathbf{p})-2 \Gamma_{2211}(-\mathbf{p}, \mathbf{p}) \\
& +2 \Gamma_{3311}(-\mathbf{p}, \mathbf{p})=0 \text {, } \\
& -s \Gamma_{232333}(\mathbf{0},-\mathbf{p}, \mathbf{p})-2 \Gamma_{2233}(-\mathbf{p}, \mathbf{p}) \\
& +2 \Gamma_{3333}(-\mathbf{p}, \mathbf{p})-\Gamma_{2323}(-\mathbf{p}, \mathbf{p})=0 \\
& -s \Gamma_{231312}(\mathbf{0},-\mathbf{p}, \mathbf{p})-2 \Gamma_{1212}(-\mathbf{p}, \mathbf{p}) \\
& +2 \Gamma_{1313}(-\mathbf{p}, \mathbf{p})=0 \text {, } \\
& -s \Gamma_{23231313}\left(\mathbf{0}, \mathbf{p}^{(1)}, \mathbf{p}^{(2)}, \mathbf{p}^{(3)}\right)-2 \Gamma_{221313}\left(\mathbf{p}^{(1)}, \mathbf{p}^{(2)}, \mathbf{p}^{(3)}\right) \\
& +2 \Gamma_{331313}\left(\mathbf{p}^{(1)}, \mathbf{p}^{(2)}, \mathbf{p}^{(3)}\right)-2 \Gamma_{122313}\left(\mathbf{p}^{(2)}, \mathbf{p}^{(1)}, \mathbf{p}^{(3)}\right) \\
& -\Gamma_{122313}\left(\mathbf{p}^{(3)}, \mathbf{p}^{(1)}, \mathbf{p}^{(2)}\right)=0 \text {, }
\end{aligned}
$$

\section{The semi-soft case}

Now we consider semi-soft NEs modeled by the elastic energy (4.1) supplemented with the explicit uniaxial term (2.11). The aligning stress $h$ breaks the reference space rotation symmetry in much the same way as the stress $\underline{\sigma}$ utilized in Appendix B 1 Thus, we basically just have to repeat the steps carried out in Appendix B1] with

$$
\begin{aligned}
\mathcal{H}_{\sigma} \rightarrow \mathcal{H}_{h, \sigma} & =\mathcal{H}_{h}-\int d^{d} x \sigma_{i j} w_{i j} \\
& =\mathcal{H}-\int d^{d} x \sigma_{h, i j} w_{i j}
\end{aligned}
$$

where

$$
\sigma_{h, i j}=\sigma_{i j}+\frac{h}{d}\left(\delta_{i 1} \delta_{j 1}+\delta_{i 2} \delta_{j 2}\right)-\frac{(d-1) h}{d} \delta_{i 3} \delta_{j 3} .
$$

This analysis leads in Fourier space to the Ward identity

$$
s \Gamma_{2323}(\mathbf{0}, \mathbf{0})-h=0
$$

provided that the equations of state $\Gamma_{22}(\mathbf{0})=0$ and $\Gamma_{33}(\mathbf{0})=0$ are satisfied. At 0-loop level the identity (B15) reduced to the relation between $h$ and $b_{5}$ stated in Eq. (4.50). 


\section{APPENDIX C: WARD IDENTITIES FOR NEMATIC ELASTOMERS II}

The mixing invariance of NEs leads to Ward identities analogous to those for CSEs derived in Appendix A Since the derivation of the 2 sets of identities is similar and Appendix $\mathrm{A}$ is fairly detailed we restrict ourselves here to mention differences in the derivations and to list results.

Introducing external fields via the step A1 we find that the free energy of NEs satisfies the identity

$$
\begin{aligned}
& F\left[h_{a}(\mathbf{x}), h_{d}(\mathbf{x})\right]=-\theta_{a} \int d^{d} x x_{a} h_{d}(\mathbf{x}) \\
& +F\left[h_{a}\left(x_{c}+\theta_{c} x_{d}, x_{d}\right), h_{d}\left(x_{c}+\theta_{c} x_{d}, x_{d}\right)+\theta_{a} h_{a}(\mathbf{x})\right] .
\end{aligned}
$$

Thus, the quantity $L_{a}=\partial F / \partial \theta_{a}$ obeys the equation

$$
\begin{aligned}
& L_{a}=-\int d^{d} x x_{a} h_{d}(\mathbf{x})+\int d^{d} x m_{d}(\mathbf{x}) h_{a}(\mathbf{x}) \\
& +\int d^{d} x m_{c}(\mathbf{x}) \frac{\partial h_{c}(\mathbf{x})}{\partial x_{a}} x_{d}+\int d^{d} x m_{d}(\mathbf{x}) \frac{\partial h_{d}(\mathbf{x})}{\partial x_{a}} x_{d} \\
& =0 .
\end{aligned}
$$

By taking the appropriate functional derivatives with respect to the order parameter, we obtain the Ward identities

$$
\begin{aligned}
& \left.i \frac{\partial}{\partial p_{a}^{(1)}} \Gamma_{d d b}^{(1,2)}\left(\mathbf{p}^{(1)}, \mathbf{p}^{(2)}, \mathbf{p}^{(3)}\right)\right|_{\mathbf{p}^{(1)}=\mathbf{0}}=\Gamma_{a b}^{(2,0)}\left(\mathbf{p}^{(2)}, \mathbf{p}^{(3)}\right) \\
& -\frac{\partial}{\partial p_{d}^{(2)}} \Gamma_{b d}^{(1,1)}\left(\mathbf{p}^{(2)}, \mathbf{p}^{(3)}\right) p_{a}^{(2)} \\
& -\frac{\partial}{\partial p_{d}^{(3)}} \Gamma_{b d}^{(1,1)}\left(\mathbf{p}^{(2)}, \mathbf{p}^{(3)}\right) p_{a}^{(3)}, \\
& \left.i \frac{\partial}{\partial p_{a}^{(1)}} \Gamma_{d d d}^{(0,3)}\left(\mathbf{p}^{(1)}, \mathbf{p}^{(2)}, \mathbf{p}^{(3)}\right)\right|_{\mathbf{p}^{(1)}=\mathbf{0}} \\
& =\Gamma_{a d}^{(1,1)}\left(\mathbf{p}^{(2)}, \mathbf{p}^{(3)}\right)+\Gamma_{a d}^{(1,1)}\left(\mathbf{p}^{(3)}, \mathbf{p}^{(2)}\right) \\
& -\frac{\partial}{\partial p_{d}^{(2)}} \Gamma_{d d}^{(0,2)}\left(\mathbf{p}^{(2)}, \mathbf{p}^{(3)}\right) p_{a}^{(2)} \\
& -\frac{\partial}{\partial p_{d}^{(3)}} \Gamma_{d d}^{(0,2)}\left(\mathbf{p}^{(2)}, \mathbf{p}^{(3)}\right) p_{a}^{(3)}, \\
& \left.i \frac{\partial}{\partial p_{a}^{(1)}} \Gamma_{d d d d}^{(0,4)}\left(\mathbf{p}^{(1)}, \mathbf{p}^{(2)}, \mathbf{p}^{(3)}, \mathbf{p}^{(4)}\right)\right|_{\mathbf{p}^{(1)}=\mathbf{0}} \\
& = \\
& \Gamma_{a d d}^{(1,2)}\left(\mathbf{p}^{(2)}, \mathbf{p}^{(3)}, \mathbf{p}^{(4)}\right)+\Gamma_{a d d}^{(1,2)}\left(\mathbf{p}^{(3)}, \mathbf{p}^{(2)}, \mathbf{p}^{(4)}\right) \\
& +\Gamma_{a d d}^{(1,2)}\left(\mathbf{p}^{(4)}, \mathbf{p}^{(2)}, \mathbf{p}^{(3)}\right) \\
& -\frac{\partial}{\partial p_{d}^{(2)}} \Gamma_{d d d}^{(0,3)}\left(\mathbf{p}^{(2)}, \mathbf{p}^{(3)}, \mathbf{p}^{(4)}\right) p_{a}^{(2)} \\
& -\frac{\partial}{\partial p_{d}^{(3)}} \Gamma_{d d d}^{(0,3)}\left(\mathbf{p}^{(2)}, \mathbf{p}^{(3)}, \mathbf{p}^{(4)}\right) p_{a}^{(3)} \\
& -\frac{\partial}{\partial p_{d}^{(4)}} \Gamma_{d d d}^{(0,3)}\left(\mathbf{p}^{(2)}, \mathbf{p}^{(3)}, \mathbf{p}^{(4)}\right) p_{a}^{(4)} .
\end{aligned}
$$

\section{APPENDIX D: ALTERNATIVE RENORMALIZATION SCHEME FOR NEMATIC ELASTOMERS}

Our renormalization scheme (4.16) which involves a re-parameterization of the coordinate $x_{d}$ follows closely the approach developed by Grinstein and Pelcovitz [22]. Though this approach has been established for more that 2 decades now, it is not clear how the known strategies of proving renormalizability apply to it. In this appendix we briefly present an alternative renormalization scheme for NEs that does not entail a re-parameterization of $x_{d}$. We demonstrate that the alternative formulation leads exactly to the same results as our original approach.

Let us revisit our Hamiltonian (4.10) as a starting point. To reduce the number of scaling variables we set $T=\check{T} K$. This step yields

$$
\begin{aligned}
\frac{\mathcal{H}}{T} & =\frac{1}{2 \check{T}} \int d^{d_{\perp}} x_{\perp} \int d x_{d}\left\{\check{\omega} v_{d d}^{2}+\left(\nabla_{\perp}^{2} u_{d}\right)^{2}\right. \\
& \left.+2 \check{g} v_{d d} v_{a a}+\check{f} v_{a a}^{2}+2 \check{h} v_{a b}^{2}\right\},
\end{aligned}
$$

where $\check{\omega}=C_{1} / K, \check{g}=C_{2} / K, \check{f}=C_{3} / K$ and $\check{h}=C_{4} / K$. Note that $\check{h}$ has no counterpart in Eq. (4.12) and that we thus have an additional parameter in comparison to Eq. (4.12). Due to this additional parameter, the Hamiltonian (D1) has an additional invariance, viz. it is invariant in from under the rescaling $x_{d} \rightarrow \beta^{-1 / 2} x_{d}$, $u_{d} \rightarrow \beta^{1 / 2} u_{d}, u_{a} \rightarrow \beta^{1} u_{a}, \check{T} \rightarrow \beta^{1 / 2} \check{T}, \check{\omega} \rightarrow \beta^{-1} \check{\omega}$, $\check{g} \rightarrow \beta^{-1} \check{g}, \check{f} \rightarrow \beta^{-1} \check{f}$ and $\check{h} \rightarrow \beta^{-1} \check{h}$ ( $\beta$-rescaling). This scaling invariance implies that the vertex functions obey the scaling from

$$
\begin{aligned}
& \Gamma^{(M, N)}\left(\left\{\mathbf{q}_{\perp}, q_{d}\right\} ; \check{T}, \check{\omega}, \check{g}, \check{f}, \check{h}\right)=\beta^{(M+1) / 2} \\
& \times \Gamma^{(M, N)}\left(\left\{\mathbf{q}_{\perp}, \beta^{1 / 2} q_{d}\right\} ; \beta^{1 / 2} \check{T}, \frac{\check{\omega}}{\beta}, \frac{\check{g}}{\beta}, \frac{\check{f}}{\beta}, \frac{\check{h}}{\beta}\right) .
\end{aligned}
$$

Equation (D2) suggest to introduce composite parameters that are invariant under the $\beta$-rescaling. Among various possibilities we choose

$$
\begin{aligned}
& t=\mu^{-\varepsilon} \check{h}^{1 / 2} \check{T}=\mu^{-\varepsilon} T \sqrt{C_{4} / K^{3}}, \\
& \omega=\check{\omega} / \check{h}=C_{1} / C_{4}, \\
& g=\check{g} / \check{h}=C_{2} / C_{4}, \\
& f=\check{f} / \check{h}=C_{3} / C_{4} .
\end{aligned}
$$

Note that these composite parameters are identical to the parameters we introduced by switching from Eq. (4.10) to 4.12).

After this prelude we specify our alternative renormalization scheme,

$$
\begin{aligned}
& \check{T} \rightarrow \check{T}=Z_{T} \check{T}, \\
& \check{\omega} \rightarrow \check{\check{\omega}}=Z_{T}^{-1} Z_{\omega} \check{\check{\omega}}, \\
& \check{g} \rightarrow \check{g}=Z_{T}^{-1} Z_{g} \check{g}, \\
& \check{f} \rightarrow \check{f}=Z_{T}^{-1} Z_{h} \check{f}, \\
& \check{h} \rightarrow \check{\check{h}}=Z_{T}^{-1} Z_{h} \check{h} .
\end{aligned}
$$


With this scheme

$$
\begin{aligned}
\frac{\mathcal{H}}{T} & \rightarrow \frac{1}{2 \check{T}} \int d^{d_{\perp}} x_{\perp} \int d x_{d}\left\{Z_{\omega} \check{\omega} v_{d d}^{2}+Z_{T}\left(\nabla_{\perp}^{2} u_{d}\right)^{2}\right. \\
& \left.+2 Z_{g} \check{g} v_{d d} v_{a a}+Z_{f} \check{f} v_{a a}^{2}+2 Z_{h} \check{h} v_{a b}^{2}\right\} .
\end{aligned}
$$

Next we derive a RGE for the vertex functions. Exploiting as usual the independence of the bare theory of $\mu$ and upon switching via $t, \omega, g$ and $f$ to the benign parameters $t, \kappa, \rho$ and $\sigma$ we get

$$
\begin{aligned}
& {\left[\mu \partial_{\mu}+\zeta \check{h} \partial_{\breve{h}}+\beta_{t} \partial_{t}+\beta_{\kappa} \partial_{\kappa}+\beta_{\rho} \partial_{\rho}+\beta_{\sigma} \partial_{\sigma}\right]} \\
& \times \Gamma^{(M, N)}\left(\left\{\mathbf{q}_{\perp}, q_{d}\right\} ; t, \kappa, \rho, \sigma, \check{h}, \mu\right)=0,
\end{aligned}
$$

where

$$
\zeta=\left.\mu \partial_{\mu} \ln \check{h}\right|_{0}=\gamma_{T}-\gamma_{h}
$$

and where the Wilson $\beta$ - and $\gamma$-functions are defined as usual. By solving the RGE (D6), supplementing the solution with a dimensional analysis and by exploiting Eq. (D2) with the choice $\beta=\check{h}$ for the parameter of the $\beta$-rescaling we find

$$
\begin{aligned}
& \Gamma^{(M, N)}\left(\left\{\mathbf{q}_{\perp}, q_{d}\right\} ; t, \kappa, \rho, \sigma, \check{h}, \mu\right)=\overline{\breve{h}}(\ell)^{(M+1) / 2} \\
& \times \Gamma^{(M, N)}\left(\left\{\frac{\mathbf{q}_{\perp}}{\mu \ell}, \frac{\bar{h}(\ell)^{1 / 2} q_{d}}{(\mu \ell)^{2}}\right\} ; \bar{t}(\ell), \bar{\kappa}(\ell), \bar{\rho}(\ell), \bar{\sigma}(\ell), 1, \mu \ell\right) .
\end{aligned}
$$

Now we take a closer look at the RG flow of $\breve{h}$. Comparing the renormalized Hamiltonians (D5) and (4.17) we learn that

$$
Z_{h}=Z_{T} Z
$$

provided the left and the right hand side are expressed in terms of the same variables, here in particular $t, \kappa, \rho$ and $\sigma$. Using Eq. (D7) and the definition of the Wilson $\gamma$-functions we find

$$
\zeta=-\gamma
$$

From the corresponding characteristics, we consequentially obtain

$$
\bar{h}(\ell) \sim \bar{Z}(\ell)^{-1} .
$$

Collecting Eqs. (D8) and (D10) we learn that our original and our alternative formulation lead to equivalent scaling results for the vertex functions. Of course, all results that follow from the scaling forms for the vertex functions, in particular the anomalous behavior of the elastic moduli, are identical for both approaches.

\section{APPENDIX E: CALCULATION OF FEYNMAN DIAGRAMS}

Here we give some details on the calculation of the Feynman diagrams listed in Figs. 11 to 3. The first part of this Appendix contains 2 representative examples. All the remaining diagrams may then be computed by similar means. The second part features 2 parameter integrals. All 2-leg diagrams can be expressed in terms of these integrals.

\section{Examples}

As a first example we consider the first diagram in the first row of Fig. 1. For the sake of the argument we refer to this diagram as $A_{d d}$. Regarding CSEs this diagram stands for the mathematical formula

$$
\begin{aligned}
A_{d d} & =q_{d}^{2} \frac{g^{2}}{2 T^{2}} \int_{\mathbf{k}} k_{a}\left(k_{a}+q_{a}\right) k_{b}\left(k_{b}+q_{b}\right) \\
& \times G_{d d}(\mathbf{k}) G_{d d}(\mathbf{k}+\mathbf{q}),
\end{aligned}
$$

where $\mathbf{q}$ is an external momentum running trough the diagram. $\int_{\mathbf{k}}$ is an abbreviation for $1 /(2 \pi)^{d} \int d^{d \perp} k_{\perp} \int d k_{d}$. Simple power counting reveals that the superficial degree of divergence of $A_{d d}$ is zero. Hence, it is sufficient to evaluate the diagram at vanishing external momentum. For $\mathbf{q}=\mathbf{0}, A_{d d}$ reduces to

$$
A_{d d}=q_{d}^{2} \frac{g^{2}}{2} B^{2} M_{42}^{(1)}
$$

with $M_{42}^{(1)}$ being a specification of the parameter integral $M_{l n}^{(1)}$ defined in Eq. (E7). Exploiting the result (E11) we obtain

$$
A_{d d}=q_{d}^{2} g^{2} \frac{B^{1 / 2}}{A^{1 / 2}} \frac{\tau^{-\varepsilon / 4}}{16 \pi \varepsilon}
$$

as the final result for $A_{d d}$. Regarding NEs we simply have to replace the $g^{2}$ stemming from the vertices by $(\omega-g)^{2}$.

As a second example we illustrate the computation of the last diagram in the row line of Fig. 11 Let us call this diagram $C_{d d}$. In case of the CSE model it visualized the formula

$$
\begin{aligned}
C_{d d} & =\frac{g^{2}}{T^{2}} \int_{\mathbf{k}} k_{d}^{2}\left[\mathbf{q}_{\perp} \cdot\left(\mathbf{q}_{\perp}+\mathbf{k}_{\perp}\right)\right]^{2} \\
& \times G_{d d}(\mathbf{k}) G_{d d}(\mathbf{k}+\mathbf{q})
\end{aligned}
$$

The superficial degree of divergence of this diagram is 2. Hence an evaluation at $\mathbf{q}=\mathbf{0}$ is not sufficient and we rather have to expand $C_{d d}$ in powers of the external momentum, Using the parameter integral $M_{l n}^{(2)}$ defined in Eq. (E12) this expansion can be written as

$$
C_{d d}=\mathbf{q}_{\perp}^{4} g^{2} B^{2}\left\{M_{02}^{(2)}-\frac{9}{2} B M_{43}^{(2)}+2 B^{2} M_{86}^{(2)}\right\}(. \mathrm{E} 5)
$$

All the other terms in this expansion turn out to be UV convergent. Using Eq. E13) the final result for $C_{d d}$ is now readily found to be

$$
C_{d d}=\mathbf{q}_{\perp}^{4} g^{2} \frac{B^{3 / 2}}{A^{3 / 2}} \frac{\tau^{-\varepsilon / 4}}{64 \pi \varepsilon} .
$$

Again, one simply has to replace the $g^{2}$ stemming from the vertices by $(\omega-g)^{2}$ if one is interested in NEs. 


\section{Parameter integrals}

Many of the 2-leg diagrams can be expressed in terms of the parameter integral

$$
M_{l n}^{(1)}=\int_{\mathbf{k}} \frac{\mathbf{k}_{\perp}^{l}}{\left[B \tau+A k_{d}^{2}+B \mathbf{k}_{\perp}^{4}\right]^{n}} .
$$

The calculation of this integral can be simplified by rescaling the momenta,

$$
M_{l n}^{(1)}=A^{-1 / 2} B^{-(d+l-1) / 4} \int_{\mathbf{k}} \frac{\mathbf{k}_{\perp}^{l}}{\left[B \tau+k_{d}^{2}+\mathbf{k}_{\perp}^{4}\right]^{n}} .
$$

Next, we employ Schwinger representation,

$$
\begin{aligned}
& M_{l n}^{(1)}=\frac{A^{-1 / 2} B^{-(d+l-1) / 4}}{(2 \pi)^{d} \Gamma(n)} \int_{0}^{\infty} d s s^{n-1} \exp (-s B \tau) \\
& \times \int d^{d_{\perp}} k_{\perp} \mathbf{k}_{\perp}^{l} \exp \left(-s \mathbf{k}_{\perp}^{4}\right) \int d k_{d} \exp \left(-s k_{d}^{2}\right) . \quad(\mathrm{E} 9)
\end{aligned}
$$

Now the momentum integrations are straightforward. We obtain

$$
\begin{aligned}
& M_{l n}^{(1)}=\frac{A^{-1 / 2} B^{-(d+l-1) / 4} \Gamma\left(\frac{d+l-1}{4}\right)}{2(4 \pi)^{d / 2} \Gamma(n) \Gamma\left(\frac{d-1}{2}\right)} \\
& \times \int_{0}^{\infty} d s \exp (-s B \tau) s^{-1+\frac{4 n-l-d-1}{4}} .
\end{aligned}
$$

Carrying out the remaining integration over the Schwinger parameter $s$ gives finally

$$
\begin{aligned}
& M_{l n}^{(1)}=\frac{A^{-1 / 2} B^{-(2 n-1) / 2} \Gamma\left(\frac{2+l-\varepsilon}{4}\right)}{2(4 \pi)^{d / 2} \Gamma(n) \Gamma\left(\frac{2-\varepsilon}{2}\right)} \\
& \times \Gamma\left(\frac{4 n-l-4+\varepsilon}{4}\right) \tau^{-\frac{4 n-l-4+\varepsilon}{4}}
\end{aligned}
$$

In addition to $M_{l n}^{(1)}$ it turns out to be convenient to introduce a second parameter integral, viz.

$$
M_{l n}^{(2)}=\int_{\mathbf{k}} \frac{k_{d}^{2} \mathbf{k}_{\perp}^{l}}{\left[B \tau+A k_{d}^{2}+B \mathbf{k}_{\perp}^{4}\right]^{n}} .
$$

This integral can be calculated by the same means as $M_{l n}^{(1)}$. We obtain the result

$$
\begin{aligned}
& M_{l n}^{(2)}=\frac{A^{-3 / 2} B^{-(2 n-3) / 2} \Gamma\left(\frac{2+l-\varepsilon}{4}\right)}{4(4 \pi)^{d / 2} \Gamma(n) \Gamma\left(\frac{2-\varepsilon}{2}\right)} \\
& \times \Gamma\left(\frac{4 n-l-8+\varepsilon}{4}\right) \tau^{-\frac{4 n-l-8+\varepsilon}{4}}
\end{aligned}
$$

[1] H. Finkelmann, H. J. Koch, and G. Rehage, Makromol. Chem. Rapid Commun. 2, 317 (1981).

[2] P. G. de Gennes in Liquid Crystals of One and TwoDimensional Order, p. 231, edited by W. Helfrich and G. Heppke (Springer, New York, 1980).

[3] E. M. Terentjev, J. Phys. Cond. Mat. 11, R239 (1999).

[4] P. G. de Gennes and J. Prost, The Physics of Liquid Crystals (Clarendon Press, Oxford, 1993).

[5] S. Chandrasekhar, Liquid Crystals (Cambridge University Press, Cambridge, 1992).

[6] S. T. Kim and H. Finkelmann, Macromol. Rapid Commun. 22, 429 (2001).

[7] R. Zentel, Angew. Chem. Adv. Mater. 101, 1437 (1989).

[8] C. N. Bowman and C. A. Guymon, MRS Bulletin, 15 (1997).

[9] Liquid Crystals in Complex Geometries, edited by G. P. Crawford and S. Zumer (Taylor and Francis, London,1996).

[10] M. Warner and E. M. Terentjev, Prog. Poly. Science 21, 853 (1996).

[11] T. C. Lubensky, R. Mukhopadhyay, L. Radzihovsky, and X. Xing, Phys. Rev. E 66, 011702 (2002).

[12] J. Küpfer and H. Finkelmann, Macromol. Chem. Phys. 195, 1353 (1994).

[13] H. Finkelmann, E. Nishikawa, G. G. Pereira, and M. Warner, Phys. Rev. Lett. 87, 015501 (2001).

[14] H. Finkelmann and H. Wermter, Polym. Mater. Sci. Eng. 82, 319 (2000).

[15] M. Hébert, R. Kant, and P.-G. de Gennes, J. Phys. I
France 7, 909 (1997).

[16] D. L. T. III, P. Keller, J. Naciri, R. Pink, H. Jeon, D. Shenoy, and B. Ratna, Macromolecules 34, 5868 (2001).

[17] L. Golubović and T. C. Lubensky, Phys. Rev. Lett. 63, 1082 (1989).

[18] Peter D. Olmsted. J. Phys. II (France), 4, 2215-2230 (1994)

[19] H. Finkelmann, I. Kundler, E. M. Terentjev, and M. Warner, J. Phys. II (France) 7, 1059 (1997).

[20] G. C. Verwey, M. Warner, and E. M. Terentjev, J. Phys. II (France) 6, 1273-1290 (1996).

[21] M. Warner, J. Mech. Phys. Solids 47, 1355 (1999).

[22] G. Grinstein and R. A. Pelcovits, Phys. Rev. Lett 47, 856 (1981); Phys. Rev. A 26, 915 (1982).

[23] See, e.g., D. J. Amit, Field Theory, the Renormalization Group, and Critical Phenomena (World Scientific, Singapore, 1984); J. Zinn-Justin, Quantum Field Theory and Critical Phenomena (Clarendon, Oxford, 1989).

[24] O. Stenull and T. C. Lubensky, Eurohys. Lett. 61, 776 (2003). See also, X. Xing and L. Radzihovsky, Eurohys. Lett. 61, 769 (2003).

[25] X. Xing and L. Radzihovsky, Phys. Rev. Lett. 90, 168301 (2003).

[26] L. D. Landau and E. M. Lifshitz, Theory of Elasticity, 3rd edition (Pergamon, New York, 1986),

[27] P. M. Chaikin and T. C. Lubensky, Principles of Condensed Matter Physics, (Cambridge University Press, Cambridge, 1995).

[28] Here and in the following we adopt Einstein's summation 
convention.

[29] Provided, of course, there are no alligning fields.

[30] For convenience, we set the Boltzmann constant equal to one.

[31] The $\left.\right|_{0}$ indicates that bare quantities are kept fixed while taking the derivatives.
[32] In our notation these shear moduli are given by $\mu_{L}=$ $\left(\omega+f+2 / d_{\perp}-2 g\right) / 4$ and $\mu=1$.

[33] X. Xing, R. Mukhopadhyay, T. C. Lubensky, and R. Radzihovsky, e-print: cond-mat/0302006 